\title{
Commutators of Higher Order Riesz Transform Associated with Schrödinger Operators
}

\author{
Yu Liu, ${ }^{1}$ Lijuan Wang, ${ }^{1}$ and Jianfeng Dong ${ }^{2}$ \\ ${ }^{1}$ School of Mathematics and Physics, University of Science and Technology Beijing, Beijing 100083, China \\ ${ }^{2}$ Department of Mathematics, Shanghai University, Shanghai 200444, China
}

Correspondence should be addressed to Yu Liu; liuyu75@pku.org.cn

Received 25 February 2013; Accepted 3 April 2013

Academic Editor: Józef Banaś

Copyright (c) $2013 \mathrm{Yu}$ Liu et al. This is an open access article distributed under the Creative Commons Attribution License, which permits unrestricted use, distribution, and reproduction in any medium, provided the original work is properly cited.

Let $L=-\Delta+V$ be a Schrödinger operator on $\mathbb{R}^{n}(n \geq 3)$, where $V \not \equiv \quad 0$ is a nonnegative potential belonging to certain reverse Hölder class $B_{s}$ for $s \geq n / 2$. In this paper, we prove the boundedness of commutators $\mathscr{R}_{b}^{H} f=b \mathscr{R}^{H} f-\mathscr{R}^{H}(b f)$ generated by the higher order Riesz transform $\mathscr{R}^{H}=\nabla^{2}(-\Delta+V)^{-1}$, where $b \in \mathrm{BMO}_{\theta}(\rho)$, which is larger than the space $\mathrm{BMO}\left(\mathbb{R}^{n}\right)$. Moreover, we prove that $\mathscr{R}_{b}^{H}$ is bounded from the Hardy space $H_{L}^{1}\left(\mathbb{R}^{n}\right)$ into weak $L_{\text {weak }}^{1}\left(\mathbb{R}^{n}\right)$.

\section{Introduction}

Let $L=-\Delta+V$ be a Schrödinger operator on $\mathbb{R}^{n}, n \geq 3$, where $V \not \equiv \quad 0$ is a nonnegative potential belonging to the reverse Hölder class $B_{s}$ for some $s \geq n / 2$. In this paper, we will consider the higher order Riesz transforms associated with the Schrödinger operator $L$ defined by $\mathscr{R}^{H} \doteq \nabla^{2} L^{-1}$ and the commutator

$$
\mathscr{R}_{b}^{H}(f)(x)=\mathscr{R}^{H}(b f)(x)-b(x) \mathscr{R}^{H} f(x), \quad x \in \mathbb{R}^{n} .
$$

We also consider its dual higher order transforms associated with the Schrödinger operator $L$ defined by $\widetilde{\mathscr{R}}^{H} \doteq L^{-1} \nabla^{2}$ and the commutator

$$
\widetilde{\mathscr{R}}_{b}^{H}(f)(x)=\widetilde{\mathscr{R}}^{H}(b f)(x)-b(x) \widetilde{\mathscr{R}}^{H} f(x), \quad x \in \mathbb{R}^{n},
$$

where $b \in \operatorname{BMO}_{\infty}(\rho)$, which is larger than the space $\mathrm{BMO}\left(\mathbb{R}^{n}\right)$.

Because the investigation of commutators of singular integral operators plays an important role in Harmonic analysis and PDE, many authors concentrate on this topic. It is well known that Coifman et al. [1] proved that $[b, T]$ is a bounded operator on $L^{p}$ for $1<p<\infty$ if and only if $b \in$ $\operatorname{BMO}\left(\mathbb{R}^{n}\right)$ when $T$ is a Calderón-Zygmund operator. See $[2,3]$ for the research development of the commutator $T_{b}$ on
Euclidean spaces $\mathbb{R}^{n}$ and [4-6] on spaces of homogeneous type.

In recent years, singular integral operators related to Schrödinger operators and their commutators have been brought to many scholars attention. See, for example, [7-19] and their references. Especially, Guo et al. [12] investigated the boundedness of the commutators $\mathscr{R}_{b}^{H}$ when $b \in \operatorname{BMO}\left(\mathbb{R}^{n}\right)$. But their method is not valid to prove the boundedness of the commutators $\mathscr{R}_{b}^{H}$ when $b \in \mathrm{BMO}_{\infty}(\rho)$. In fact, since

$$
\begin{aligned}
\mathscr{R}^{H} & =\nabla^{2}(-\Delta)^{-1}(-\Delta)(-\Delta+V)^{-1} \\
& =\nabla^{2}(-\Delta)^{-1}\left(I-V(-\Delta+V)^{-1}\right),
\end{aligned}
$$

then $\mathscr{R}_{b}^{H}$ may be written as follows:

$$
\mathscr{R}_{b}^{H}(f)=\left[b, T_{1} T_{2}\right] f=\left[b, T_{1}\right]\left(T_{2} f\right)+T_{1}\left[b, T_{2}\right] f,
$$

where $T_{1}=\nabla^{2}(-\Delta)^{-1}$ and $T_{2}=I-V(-\Delta+V)^{-1}$. If $b \in$ $\operatorname{BMO}\left(\mathbb{R}^{n}\right)$, by using Corollary 1 in $[12]$, we obtain the $L^{p}$ boundedness of $\mathscr{R}_{b}^{H}$. But if $b \in \operatorname{BMO}_{\theta}(\rho)$ and $b \notin \operatorname{BMO}\left(\mathbb{R}^{n}\right)$, it follows from [1] that $\left[b, T_{1}\right]$ is not bounded on $L^{p}$, and then we cannot obtain the $L^{p}$ boundedness of $\mathscr{R}_{b}^{H}$.

Motivated by $[12,15,17]$, our aim in this paper is to investigate the $L^{p}$ estimates and endpoint estimates for $\mathscr{R}_{b}^{H}$ when $b \in \operatorname{BMO}_{\infty}(\rho)$. Different from the classical higher order Riesz 
transform, there exist some new problems for the higher order Riesz transform $\mathscr{R}^{H}$. We need to obtain some new estimates for $\mathscr{R}^{H}$ when the potential $V$ satisfies more stronger conditions.

A nonnegative locally $L^{s}$-integrable function $V(1<s<$ $\infty$ ) is called to belong to $B_{s}$ if there exists a constant $C>0$ such that the reverse Hölder inequality

$$
\left(\frac{1}{|B(x, r)|} \int_{B(x, r)} V^{s} d y\right)^{1 / s} \leq C\left(\frac{1}{|B(x, r)|} \int_{B(x, r)} V d y\right)
$$

holds for every ball $B$ in $\mathbb{R}^{n}$.

Moreover, a locally bounded nonnegative function $V \in$ $B_{\infty}$, if there exists a positive constant $C$ such that

$$
\|V\|_{L^{\infty}(B(x, r))} \leq \frac{C}{|B(x, r)|} \int_{B(x, r)} V(y) d y
$$

holds for every $B(x, r)$ in $\mathbb{R}^{n}$ and $0<r<\infty$.

Obviously, $B_{s_{2}} \subset B_{s_{1}}$, if $s_{2}>s_{1}$. But it is important that the $B_{s}$ class has a property of "self-improvement"; that is, if $V \in B_{s}$, then $V \in B_{s+\varepsilon}$ for some $\varepsilon>0$. Furthermore, it is easy to see that $B_{\infty} \subseteq B_{s}$ for any $1<s<\infty$.

Assume that $V \geq 0$ and $V \in L_{\text {loc }}^{n / 2}\left(\mathbb{R}^{n}\right)$. The Schrödinger operator $L=-\Delta+V$ generates a $\left(C_{0}\right)$ semigroup $\left\{T_{t}^{L}\right\}_{t>0}=$ $\left\{e^{-t L}\right\}_{t>0}$. The maximal function with respect to the semigroup $\left\{T_{t}^{L}\right\}_{t>0}$ is given by

$$
M^{L} f(x)=\sup _{t>0}\left|T_{t}^{L} f(x)\right| .
$$

The Hardy space $H_{L}^{1}\left(\mathbb{R}^{n}\right)$ associated with the Schrödinger operator $L$ is defined as follows in terms of the maximal function mentioned earlier (cf. [20]).

Definition 1. A function $f \in L^{1}\left(\mathbb{R}^{n}\right)$ is said to be in $H_{L}^{1}\left(\mathbb{R}^{n}\right)$ if the semigroup maximal function $M^{L} f$ belongs to $L^{1}\left(\mathbb{R}^{n}\right)$. The norm of such a function is defined by

$$
\|f\|_{H_{L}^{1}}=\left\|M^{L} f\right\|_{L^{1}}
$$

We introduce the auxiliary function $\rho(x, V)=\rho(x)$ defined by

$$
\begin{aligned}
\rho(x) & =\frac{1}{m(x, V)} \\
& \doteq \sup _{r>0}\left\{r: \frac{1}{r^{n-2}} \int_{B(x, r)} V(y) d y \leq 1\right\}, \quad x \in \mathbb{R}^{n} .
\end{aligned}
$$

It is known that $0<\rho(x)<\infty$ for any $x \in \mathbb{R}^{n}$ (from Lemma 8 in Section 2).

Definition 2. Let $1<q \leq \infty$. A measurable function $a$ is

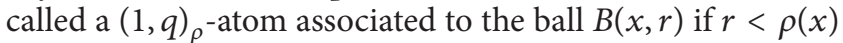
and the following conditions hold:

(i) supp $a \subset B(x, r)$ for some $x \in \mathbb{R}^{n}$ and $r>0$, (ii) $\|a\|_{L^{q\left(\mathbb{R}^{n}\right)}} \leq|B(x, r)|^{1 / q-1}$,

(iii) when $r<\rho(x) / 4, \int_{\mathbb{R}^{n}} a(x) d x=0$.

The space $H_{L}^{1}\left(\mathbb{R}^{n}\right)$ admits the following atomic decompositions (cf. [21]).

Proposition 3. Let $f \in L^{1}\left(\mathbb{R}^{n}\right)$. Then, $f \in H_{L}^{1}\left(\mathbb{R}^{n}\right)$ if and only if $f$ can be written as $f=\sum_{j} \lambda_{j} a_{j}$, where $a_{j}$ are $(1, q)_{\rho}$-atoms and $\sum_{j}\left|\lambda_{j}\right|<\infty$. Moreover,

$$
\|f\|_{H_{L}^{1}} \sim \inf \left\{\sum_{j}\left|\lambda_{j}\right|\right\}
$$

where the infimum is taken over all atomic decompositions of $f$ into $H_{L}^{1}$-atoms.

Following [17], the class $\mathrm{BMO}_{\theta}(\rho)$ of locally integrable function $b$ is defined as follows:

$$
\frac{1}{|B(x, r)|} \int_{B(x, r)}\left|b(y)-b_{B}\right| d y \leq C\left(1+\frac{r}{\rho(x)}\right)^{\theta},
$$

for all $x \in \mathbb{R}^{n}$ and $r>0$, where $\theta>0$ and $b_{B}=$ $(1 /|B|) \int_{B} b(y) d y$. A norm for $b \in \mathrm{BMO}_{\theta}(\rho)$, denoted by $[b]_{\theta}$, is given by the infimum of the constants satisfying (11), after identifying functions that differ upon a constant. If we let $\theta=0$ in (11), then $\operatorname{BMO}_{\theta}(\rho)$ is exactly the John-Nirenberg space $\mathrm{BMO}\left(\mathbb{R}^{n}\right)$. Denote that $\mathrm{BMO}_{\infty}(\rho)=\bigcup_{\theta>0} \mathrm{BMO}_{\theta}(\rho)$. It is easy to see that $\mathrm{BMO}\left(\mathbb{R}^{n}\right) \subset \mathrm{BMO}_{\theta}(\rho) \subset \mathrm{BMO}_{\theta^{\prime}}(\rho)$ for $0<\theta \leq \theta^{\prime}$. Bongioanni et al. [17] gave some examples to clarify that the space $\operatorname{BMO}\left(\mathbb{R}^{n}\right)$ is a subspace of $\operatorname{BMO}_{\infty}(\rho)$.

Let $\rho_{1}(x)$ be the auxiliary function of $|\nabla V(x)|$. Our main results are given as follows.

Theorem 4. Suppose that $V \in B_{s}$ for some $s \geq n,|\nabla V| \in B_{s_{1}}$ $\left(s_{1} \geq n / 2\right), \rho(x) \lesssim \rho_{1}(x)$, and $\rho(x) \lesssim 1$. Let $b \in B M O_{\infty}(\rho)$. The commutator $\widetilde{\mathscr{R}}_{b}^{H}(f)$ is bounded on $L^{p}\left(\mathbb{R}^{n}\right)$ for $s_{1}^{\prime}<p<$ $\infty$, where $\left(1 / s_{1}\right)+\left(1 / s_{1}^{\prime}\right)=1$.

By duality, we immediately have the following.

Corollary 5. Suppose that $V \in B_{s}$ for some $s \geq n,|\nabla V| \in B_{s_{1}}$ $\left(s_{1} \geq n / 2\right), \rho(x) \lesssim \rho_{1}(x)$, and $\rho(x) \lesssim 1$. Let $b \in B M O_{\infty}(\rho)$. The commutator $\mathscr{R}_{b}^{H}$ is bounded on $L^{p}\left(\mathbb{R}^{n}\right)$ for $1<p<s_{1}$.

Furthermore, we get the endpoint estimate for the commutator $\mathscr{R}_{b}^{H}$.

Theorem 6. Suppose that $V \in B_{s}$ for some $s \geq n,|\nabla V| \in B_{s_{1}}$ $\left(s_{1} \geq n / 2\right), \rho(x) \lesssim \rho_{1}(x)$, and $\rho(x) \lesssim 1$. Let $b \in B M O_{\infty}(\rho)$. Then, for any $\lambda>0$,

$$
\begin{array}{r}
\left|\left\{x \in \mathbb{R}^{n}:\left|\mathscr{R}_{b}^{H}(f)(x)\right|>\lambda\right\}\right| \lesssim \frac{[b]_{\theta}}{\lambda}\|f\|_{H_{L}^{1}\left(\mathbb{R}^{n}\right)}, \\
\forall f \in H_{L}^{1}\left(\mathbb{R}^{n}\right) .
\end{array}
$$

Namely, the commutator $\mathscr{R}_{b}^{H}$ is bounded from $H_{L}^{1}\left(\mathbb{R}^{n}\right)$ into $L_{\text {weak }}^{1}\left(\mathbb{R}^{n}\right)$. 
This paper is organized as follows. In Section 2, we collect some known facts about the auxiliary function $\rho(x)$ and some necessary estimates for the kernel of the higher order Riesz transform $\mathscr{R}^{H}$. In Section 3, we give the proof of Theorems 4 and 6 . Section 4 gives the corresponding results when the potential $V$ satisfies stronger conditions. In Section 5 , we give some examples for the potentials $V$ in Theorems 4 and 6 .

Throughout this paper, unless otherwise indicated, we always assume that $0 \not \equiv V \in B_{s}$ for some $s>n$. We will use $C$ to denote the positive constants, which are not necessarily same at each occurrence even be different in the same line, and may depend on the dimension $n$ and the constant in (5) or (6). By $A \sim B$ and $A \leqslant B$, we mean that there exist some constants $C, C^{\prime}$ such that $1 / C \leq A / B \leq C$ and $A \leq C^{\prime} B$, respectively.

\section{Some Lemmas}

In this section, we collect some known results about auxiliary function $\rho(x)$ and some necessary estimates for the kernel of the higher order Riesz transform in the paper.

Lemma 7. $V \in B_{s}(s \geq n / 2)$ is a doubling measure; that is, there exists a constant $C>0$ such that

$$
\int_{B(x, 2 r)} V(y) d y \leq C \int_{B(x, r)} V(y) d y .
$$

Especially, there exist constants $\mu \geq 1$ and $C$ such that

$$
\int_{B(x, t r)} V(y) d y \leq C t^{n \mu} \int_{B(x, r)} V(y) d y
$$

holds for every ball $B(x, r)$ and $t>1$.

Lemma 8. There exist constants $C, k_{0}>0$ such that

$$
\frac{1}{C}\left(1+\frac{|x-y|}{\rho(x)}\right)^{-k_{0}} \leq \frac{\rho(y)}{\rho(x)} \leq C\left(1+\frac{|x-y|}{\rho(x)}\right)^{k_{0} /\left(k_{0}+1\right)} .
$$

In particular, $\rho(y) \sim \rho(x)$ if $|x-y|<C \rho(x)$.

Using the Hölder inequality and $B_{s}$ condition, we have the following.

Lemma 9. Let

$$
\int_{B(x, R)} \frac{V(y) d y}{|x-y|^{n-2}} \leq \frac{C}{R^{n-2}} \int_{B(x, R)} V(y) d y .
$$

Moreover, if $V \in B_{n}$, then there exists $C>0$ such that

$$
\int_{B(x, R)} \frac{V(y) d y}{|x-y|^{n-1}} \leq \frac{C}{R^{n-1}} \int_{B(x, R)} V(y) d y .
$$

Lemma 10. (1) For $0<r<R<\infty$,

$$
\begin{gathered}
\frac{1}{r^{n-2}} \int_{B(x, r)} V(y) d y \leq C\left(\frac{r}{R}\right)^{2-n / s} \frac{1}{R^{n-2}} \int_{B(x, R)} V(y) d y, \\
\frac{1}{r^{n-2}} \int_{B(x, r)} V(y) d y \sim 1 \quad \text { iff } r \sim \rho(x) .
\end{gathered}
$$

(2) There exist $C>0$ and $k_{0}^{\prime}>0$ such that

$$
\frac{1}{R^{n-2}} \int_{B(x, R)} V(y) d y \leq C\left(1+\frac{R}{\rho(x)}\right)^{k_{0}^{\prime}} .
$$

Let $\Gamma(x, y)$ be the fundamental solution of $L$. Then, there exists $C_{l}>0$ such that for each $l>0$,

$$
|\Gamma(x, y)| \leq \frac{C_{l}}{(1+|x-y| / \rho(x))^{l}} \frac{1}{|x-y|^{n-2}} .
$$

In particular, $\Gamma(x, y)=\Gamma(x, y, 0)=\Gamma(y, x, 0)$ is the fundamental solution of the Schrödinger operator $L$. If $V \in B_{n}$, then there exists $C_{l}>0$ such that for each $l>0$,

$$
|\nabla \Gamma(x, y)| \leq \frac{C_{l}}{(1+|x-y| / \rho(x))^{l}} \frac{1}{|x-y|^{n-1}} .
$$

The previous facts had been obtained by Shen in [8].

We denote the fundamental solution of $-\Delta$ by $\Gamma_{0}(x, y)$, which satisfies the following.

(i) There exists $C>0$ such that

$$
\left|\Gamma_{0}(x, y)\right| \leq \frac{C}{|x-y|^{n-2}} .
$$

(ii) There exists $C>0$ such that

$$
\left|\nabla \Gamma_{0}(x, y)\right| \leq \frac{C}{|x-y|^{n-1}} .
$$

Lemma 11. Suppose that $V \in B_{s}$ for somes $>$ nand $|\nabla V| \in B_{s_{1}}$ for some $s_{1}>n / 2$. Assume that $(-\Delta+V) u=0$ in $B\left(x_{0}, 2 R\right)$. Then,

$$
\begin{aligned}
\left|\nabla^{2} u(x)\right| \lesssim & \left(1+\frac{R}{\rho\left(x_{0}\right)}\right)^{k_{0}^{\prime}} \\
& \times \sup _{B\left(x_{0}, 2 R\right)}|u(y)|\left(\int_{B\left(x_{0}, 2 R\right)} \frac{|\nabla V(y)|}{|x-y|^{n-1}} d y+\frac{1}{R^{2}}\right) \\
& +\frac{1}{R}\left(1+\frac{R}{\rho\left(x_{0}\right)}\right)^{k_{0}^{\prime}} \sup _{B\left(x_{0}, 2 R\right)}|\nabla u(y)| .
\end{aligned}
$$

Proof. Let $\phi \in C_{c}^{\infty}\left(B\left(x_{0}, 2 R\right)\right)$ such that $\phi \equiv 1$ on $B\left(x_{0}\right.$, $(3 / 2) R), 0<\phi \leq 1,|\nabla \phi| \leq C R^{-1},\left|\nabla^{2} \phi\right| \leq C R^{-2}$, and $\left|\nabla^{3} \phi\right| \leq C R^{-3}$. Since

$$
u(x) \phi(x)=\int_{\mathbb{R}^{n}} \Gamma_{0}(x, y)(-\Delta)(u \phi)(y) d y,
$$

then, for $x \in B\left(x_{0}, R\right)$, 


$$
\begin{aligned}
& \nabla^{2} u(x) \\
&= \int_{\mathbb{R}^{n}} \nabla \Gamma_{0}(x, y)(-\Delta)(\nabla(u \phi))(y) d y \\
&= \int_{\mathbb{R}^{n}} \nabla \Gamma_{0}(x, y)(-\Delta)(\nabla u \phi+u \nabla \phi)(y) d y \\
&= \int_{\mathbb{R}^{n}} \nabla \Gamma_{0}(x, y)\left(-V(y) u(y) \nabla \phi(y)-2 \nabla u(y) \cdot \nabla^{2} \phi(y)\right. \\
&\quad-u(y) \Delta \nabla \phi(y)) d y \\
& \quad+\int_{\mathbb{R}^{n}} \nabla \Gamma_{0}(x, y)(-\nabla V u \phi-V \nabla u \phi) d y .
\end{aligned}
$$

Therefore, we have, for $x \in B\left(x_{0}, R\right)$,

$$
\begin{aligned}
& \left|\nabla^{2} u(x)\right| \\
& \leq \int_{B\left(x_{0}, 2 R\right)} \frac{|\nabla V(y)||u(y)|}{|x-y|^{n-1}} d y+\int_{B\left(x_{0}, 2 R\right)} \frac{V(y)|\nabla u(y)|}{|x-y|^{n-1}} d y \\
& \quad+\frac{1}{R^{n}} \int_{B\left(x_{0}, 2 R\right)}|V(y) u(y)| d y+\frac{1}{R^{n+1}} \int_{B\left(x_{0}, 2 R\right)}|\nabla u(y)| d y \\
& \quad+\frac{1}{R^{n+2}} \int_{B\left(x_{0}, 2 R\right)}|u(y)| d y \\
& \leq \sup _{B\left(x_{0}, 2 R\right)}|u(y)|\left(\int_{B\left(x_{0}, 2 R\right)} \frac{|\nabla V(y)|}{|x-y|^{n-1}} d y\right. \\
& \quad+\sup _{B\left(x_{0}, 2 R\right)}|\nabla u(y)|\left(\int_{B\left(x_{0}, 2 R\right)} \frac{|V(y)|}{|x-y|^{n-1}} d y+\frac{1}{R}\right) \\
& \leq\left(1+\frac{R}{\rho\left(x_{0}\right)}\right)^{R_{0}^{\prime}} \sup _{B\left(x_{0}, 2 R\right)}|u(y)| \\
& \quad \times\left(\int_{B\left(x_{0}, 2 R\right)} \frac{|\nabla V(y)|}{|x-y|^{n-1}} d y+\frac{1}{R^{2}}\right) \\
& +\frac{1}{R}\left(1+\frac{R}{\rho\left(x_{0}\right)}\right)^{k_{0}^{\prime}} \sup _{B\left(x_{0}, 2 R\right)}|\nabla u(y)|, \\
& \quad\left(\int^{2}\right)
\end{aligned}
$$

where we use Lemma 9 and (2) in Lemma 10 in the last step.

Therefore, we complete the proof of the lemma.

Furthermore, we get the following corollary via the proof of Lemma 11.

Corollary 12. Suppose that $V \in B_{s}$ for some $s>n$ and $|\nabla V| \in$ $B_{s_{1}}$ for some $s_{1}>n / 2$. There exists a constant $C_{l}>0$ such that for each $l>0$,

$$
\begin{aligned}
\left|\nabla^{2} \Gamma(x, y)\right| \leq & \frac{C_{l}}{(1+|x-y| / \rho(x))^{l}} \frac{1}{|x-y|^{n-2}} \\
& \times\left(\int_{B(x, 2|x-y|)} \frac{|\nabla V(z)|}{|x-y|^{n-1}} d z+\frac{1}{|x-y|^{2}}\right) .
\end{aligned}
$$

Lemma 13. Suppose that $V \in B_{s}$ for some $s>n$ and $|\nabla V| \in$ $B_{s_{1}}$ for some $s_{1}>n / 2$. There exists a constant $C_{l}>0$ such that for each $l>0$,

$$
\begin{aligned}
& \left|\nabla_{y}^{2} \Gamma(x+h, y)-\nabla_{y}^{2} \Gamma(x, y)\right| \\
& \leq \frac{C_{l}}{(1+|x-y| / \rho(x))^{l}} \frac{|h|^{\delta}}{|x-y|^{n-2+\delta}} \\
& \quad \times\left(\int_{B(x, 2|x-y|)} \frac{|\nabla V(z)|}{|x-z|^{n-1}} d z+\frac{1}{|x-y|^{2}}\right),
\end{aligned}
$$

where $\delta=1-n / t>0$ for $t>n$.

Proof. Let $R=|x-y| / 4$. Assume that $|h|<R / 2$. It follows from the embedding theorem of Morrey, Corollary 1, and Remark 4.9 in [8] that

$$
\begin{aligned}
& \left|\nabla_{y}^{2} \Gamma(x+h, y)-\nabla_{y}^{2} \Gamma(x, y)\right| \\
& \leq C|h|^{1-n / t}\left(\int_{B(x, R)}\left|\nabla_{x} \nabla_{y}^{2} \Gamma(z, y)\right|^{t} d z\right)^{1 / t} \\
& \leq C\left(\frac{|h|}{R}\right)^{1-n / t}(1+R m(x, V))^{k_{0}} \sup _{B(x, 2 R)}\left|\nabla_{y}^{2} \Gamma(z, y)\right| \\
& \leq \frac{C_{l}}{(1+|x-y| / \rho(x))^{l}} \frac{|h|^{\delta}}{|x-y|^{n-2+\delta}} \\
& \quad \times\left(\int_{B(x, 2|x-y|)} \frac{|\nabla V(z)|}{|x-z|^{n-1}} d z+\frac{1}{|x-y|^{2}}\right),
\end{aligned}
$$

where $\delta=1-n / t>0$.

Similarly, we have the following.

Lemma 14. Suppose that $V \in B_{s}$ for some $s>n$ and $|\nabla V| \in$ $B_{s_{1}}$ for some $s_{1}>n / 2$. There exists a constant $C_{l}>0$ such that for each $l>0$,

$$
\begin{aligned}
& \left|\nabla_{x}^{2} \Gamma(x, y+h)-\nabla_{x}^{2} \Gamma(x, y)\right| \\
& \leq \frac{C_{l}}{(1+|x-y| / \rho(x))^{l}} \frac{|h|^{\delta}}{|x-y|^{n-2+\delta}} \\
& \quad \times\left(\int_{B(x, 2|x-y|)} \frac{|\nabla V(z)|}{|x-z|^{n-1}} d z+\frac{1}{|x-y|^{2}}\right),
\end{aligned}
$$

where $\delta=1-n / t>0$ for $t>n$. 
Corollary 15. Suppose that $V \in B_{s}$ for some $s>n,|\nabla V| \in B_{s_{1}}$ for some $s_{1}>n / 2$, and there exists a constant $C$ such that

$$
|\nabla V(x)| \leq C m(x, V)^{3} .
$$

There exists a constant $C_{l}>0$ such that for each $l>0$,

$$
\left|\nabla^{2} \Gamma(x, y)\right| \leq \frac{C_{l}}{(1+|x-y| / \rho(x))^{l}} \frac{1}{|x-y|^{n}} .
$$

Proof. Since $|\nabla V(z)| \leq C m(z, V)^{3}$ for $z \in B(x, 2|x-y|)$, then by using Lemma 8 ,

$$
\begin{aligned}
m(z, V) & \lesssim(1+|x-z| m(x, V))^{k_{0}} m(x, V) \\
& \lesssim(1+|x-y| m(x, V))^{k_{0}} m(x, V) .
\end{aligned}
$$

Therefore, by Corollary 12,

$$
\begin{aligned}
\left|\nabla^{2} \Gamma(x, y)\right| & \frac{C_{l}}{(1+|x-y| / \rho(x))^{l}} \frac{1}{|x-y|^{n-2}} \\
& \times\left(\int_{B(x, 2|x-y|)} \frac{|\nabla V(z)|}{|x-z|^{n-1}} d z+\frac{1}{|x-y|^{2}}\right) \\
\leqslant & \frac{C_{l}}{(1+|x-y| / \rho(x))^{l}} \frac{(1+|x-y| m(x, V))^{3 k_{0}} m(x, V)^{3}}{|x-y|^{n-2}} \\
& \times\left(\int_{B(x, 2|x-y|)} \frac{1}{|x-z|^{n-1}} d z+\frac{1}{|x-y|^{2}}\right) \\
& \leqslant \frac{C_{l}}{(1+|x-y| / \rho(x))^{l}} \frac{(1+|x-y| m(x, V))^{3 k_{0}+3}}{|x-y|^{n+1}} \\
& \times\left(\int_{B(x, 2|x-y|)} \frac{1}{|x-z|^{n-1}} d z+\frac{1}{|x-y|^{2}}\right) \\
\leqslant & C_{l} \\
& (1+|x-y| / \rho(x))^{l-3 k_{0}-3} \frac{1}{|x-y|^{n}} .
\end{aligned}
$$

Furthermore, we obtain the following corollary by using Corollary 12 and Lemma 14.

Corollary 16. Suppose that $V \in B_{s}$ for some $s>n,|\nabla V| \in B_{s_{1}}$ for some $s_{1}>n / 2$ and satisfies (32). There exists a constant $C_{l}>0$ such that for each $l>0$,

$$
\begin{aligned}
& \left|\nabla_{x}^{2} \Gamma(x, y+h)-\nabla_{x}^{2} \Gamma(x, y)\right| \\
& \quad \leq \frac{C_{l}}{(1+|x-y| / \rho(x))^{l}} \frac{|h|^{\delta}}{|x-y|^{n+\delta}}, \\
& \left|\nabla_{x}^{2} \Gamma(x+h, y)-\nabla_{x}^{2} \Gamma(x, y)\right|
\end{aligned}
$$

$$
\leq \frac{C_{l}}{(1+|x-y| / \rho(x))^{l}} \frac{|h|^{\delta}}{|x-y|^{n+\delta}}
$$

where $\delta=1-n / t>0$ for $t>n$.

Remark 17. Following Remark 5 in [22], we know that if $V$ is a nonnegative polynomial, condition (32) holds true. Therefore, Corollaries 15 and 16 also hold true.

Lemma 18. Suppose that $V \in B_{s}$ for some $s>n$, and $(1 / s)+$ $\left(1 / s^{\prime}\right)=1$.

(1) $L^{-1}(-\Delta)$ and $L^{-1} V$ are bounded on the space $L^{p}\left(\mathbb{R}^{n}\right)$, where $1 \leq p \leq s$.

(2) $\mathscr{R}^{H}$ is bounded on the space $L^{p}\left(\mathbb{R}^{n}\right)$ for $1<p \leq s$.

(3) $\widetilde{\mathscr{R}}^{H}$ is bounded on the space $L^{p}\left(\mathbb{R}^{n}\right)$ for $s^{\prime}<p<\infty$.

Since $\mathscr{R}^{H}=\nabla^{2}(-\Delta)^{-1}(-\Delta)(-\Delta+V)^{-1}$, by using (1) in Lemma 18 , we obtain the following.

Lemma 19. Suppose that $V \in B_{s}$ for some $s>n$. Then, for any $\lambda>0$,

$$
\begin{array}{r}
\left|\left\{x \in \mathbb{R}^{n}:\left|\mathscr{R}^{H}(f)(x)\right|>\lambda\right\}\right| \leq \frac{C}{\lambda}\|f\|_{L^{1}\left(\mathbb{R}^{n}\right)}, \\
\forall f \in L^{1}\left(\mathbb{R}^{n}\right) .
\end{array}
$$

2.1. Some Lemmas Related to BMO Spaces $B M O_{\theta}(\rho)$. In this section, we recall some propositions and lemmas for the BMO spaces $\operatorname{BMO}_{\theta}(\rho)$ in [17].

A ball $B(x, \rho(x))$ is called critical. In [20], Dziubański and Zienkiewicz gave the following covering lemma on $\mathbb{R}^{n}$.

Proposition 20. There exists a sequence of points $\left\{x_{k}\right\}_{k=1}^{\infty}$ in $\mathbb{R}^{n}$, such that the family of critical balls $Q_{k}=B\left(x_{k}, \rho\left(x_{k}\right)\right)$, $k \geq 1$, satisfies the following:

(i) $\bigcup_{k} Q_{k}=\mathbb{R}^{n}$;

(ii) there exists $N=N(\rho)$ such that for every $k \in \mathbb{N}$, $\operatorname{card}\left\{j: 4 Q_{j} \cap 4 Q_{k} \neq \emptyset\right\} \leq N$.

Lemma 21. Let $\theta>0$ and $1 \leq p<\infty$. If $b \in B M O_{\theta}(\rho)$, then

$$
\left(\frac{1}{|B(x, r)|} \int_{B(x, r)}\left|b(y)-b_{B}\right|^{p} d y\right)^{1 / p} \leq C[b]_{\theta}\left(1+\frac{r}{\rho(x)}\right)^{\theta^{\prime}},
$$

for all $B=B(x, r)$, with $x \in \mathbb{R}^{n}$ and $r>0$, where $\theta^{\prime}=\left(1+k_{0}\right) \theta$ and $k_{0}$ is the constant appearing in Lemma 8.

Lemma 22. Let $b \in B M O_{\theta}(\rho), B=B\left(x_{0}, r\right)$, and $p \geq 1$. Then,

$$
\left(\frac{1}{\left|2^{k} B\right|} \int_{2^{k} B}\left|b(y)-b_{B}\right|^{p} d y\right)^{1 / p} \leq C[b]_{\theta} k\left(1+\frac{2^{k} r}{\rho\left(x_{0}\right)}\right)^{\theta^{\prime}},
$$

for all $k \in \mathbb{N}$, with $\theta^{\prime}$ as in (38). 
Given that $\alpha>0$, we define the following maximal functions for $g \in L_{\text {loc }}^{1}\left(\mathbb{R}^{n}\right)$ and $x \in \mathbb{R}^{n}$ :

$$
\begin{gathered}
M_{\rho, \alpha} g(x)=\sup _{x \in B \in \mathscr{S}_{\rho, \alpha}} \frac{1}{|B|} \int_{B}|g|, \\
M_{\rho, \alpha}^{\sharp} g(x)=\sup _{x \in B \in \mathscr{R}_{\rho, \alpha}} \frac{1}{|B|} \int_{B}\left|g-g_{B}\right|,
\end{gathered}
$$

where $\mathscr{B}_{\rho, \alpha}=\left\{B(y, r): y \in \mathbb{R}^{n}, r \leq \alpha \rho(y)\right\}$.

Also, given a ball $Q \subset \mathbb{R}^{n}$, for $g \in L_{\text {loc }}^{1}(Q)$ and $x \in Q$, we define

$$
\begin{gathered}
M_{\mathrm{Q}} g(x)=\sup _{x \in B \in \mathscr{F}(\mathrm{Q})} \frac{1}{|B \cap Q|} \int_{B \cap Q}|g|, \\
M_{\mathrm{Q}}^{\sharp} g(x)=\sup _{x \in B \in \mathscr{F}(\mathrm{Q})} \frac{1}{|B \cap Q|} \int_{B \cap Q}\left|g-g_{B}\right|,
\end{gathered}
$$

where $\mathscr{F}(Q)=\{B(y, r): y \in Q, r>0\}$.

Lemma 23. For $1<p<\infty$, there exist $\beta$ and $\gamma$ such that if $\left\{Q_{k}\right\}_{k=1}^{\infty}$ is a sequence of balls as in Proposition 20, then

$$
\begin{aligned}
\int_{\mathbb{R}^{n}}\left|M_{\rho, \beta}(g)\right|^{p} \leq C( & \int_{\mathbb{R}^{n}}\left|M_{\rho, \gamma}^{\sharp}(g)\right|^{p} \\
& \left.+\sum_{k}\left|Q_{k}\right|\left(\frac{1}{\left|Q_{k}\right|} \int_{2 Q_{k}}|g|\right)\right)^{p},
\end{aligned}
$$

for all $g \in L_{\text {loc }}^{1}\left(\mathbb{R}^{n}\right)$.

\section{Proofs of the Main Results}

Firstly, in order to prove Theorem 4, we need the following lemmas. As usual, for $f \in L_{\text {loc }}^{1}\left(\mathbb{R}^{n}\right)$, we denote by $M_{p}$ the $p$-maximal function which is defined as

$$
M_{p} f(x)=\sup _{r>0}\left(\frac{1}{|B(x, r)|} \int_{B(x, r)}|f(y)|^{p} d y\right)^{1 / p} .
$$

Lemma 24. Suppose that $V \in B_{s}$ for some $s \geq n,|\nabla V| \in B_{s_{1}}$ $\left(s_{1} \geq n / 2\right), \rho(x) \leqslant \rho_{1}(x)$, and $\rho(x) \leqslant 1$. Let $b \in B M O_{\theta}(\rho)$. Then, there exists a constant $C$ such that

$$
\frac{1}{|Q|} \int_{Q}\left|\widetilde{R}_{b}^{H} f(y)\right| d y \leq C[b]_{\theta} \inf _{y \in Q} M_{p} f(y),
$$

for all $f \in L_{l o c}^{p}\left(\mathbb{R}^{n}\right)$ for $p>s_{1}^{\prime}$ and every ball $Q=B\left(x_{0}, \rho\left(x_{0}\right)\right)$. Proof. Let $f \in L^{p}\left(\mathbb{R}^{n}\right)$ and $Q=B\left(x_{0}, \rho\left(x_{0}\right)\right)$. Write $\widetilde{\mathscr{R}}_{b}^{H} f$ as

$$
\widetilde{\mathscr{R}}_{b}^{H} f=\left(b-b_{\mathrm{Q}}\right) \widetilde{\mathscr{R}}^{H} f-\widetilde{\mathscr{R}}^{H}\left(f\left(b-b_{\mathrm{Q}}\right)\right) .
$$

Firstly, by the Hölder inequality with $p>s_{1}^{\prime}$ and Lemma 21,

$$
\begin{aligned}
& \frac{1}{|Q|} \int_{Q}\left|\left(b-b_{Q}\right) \widetilde{\mathscr{R}}^{H} f(y)\right| d y \\
& \quad \leq\left(\frac{1}{|Q|} \int_{Q}\left|b-b_{\mathrm{Q}}\right|^{p^{\prime}} d y\right)^{1 / p^{\prime}}\left(\frac{1}{|Q|} \int_{\mathrm{Q}}\left|\widetilde{\mathscr{R}}^{H} f(y)\right|^{p} d y\right)^{1 / p} \\
& \quad \leq C[b]_{\theta}\left(\frac{1}{|Q|} \int_{Q}\left|\widetilde{\mathscr{R}}^{H} f(y)\right|^{p} d y\right)^{1 / p} .
\end{aligned}
$$

If we write $f=f_{1}+f_{2}$ with $f_{1}=f \chi_{2 Q}$, then

$$
\begin{aligned}
\left(\frac{1}{|Q|} \int_{Q}\left|\widetilde{R}^{H} f_{1}(y)\right|^{p} d y\right)^{1 / p} & \leq C\left(\frac{1}{|Q|} \int_{2 Q}|f(y)|^{p} d y\right)^{1 / p} \\
& \leq C \inf _{y \in Q} M_{p} f(y),
\end{aligned}
$$

where we use the fact that $\widetilde{\mathscr{R}}^{H}$ is bounded on $L^{p}\left(\mathbb{R}^{n}\right)$ with $s^{\prime}<s_{1}^{\prime}<p<\infty$.

By Corollary 12 and the Hölder inequality, we have

$$
\begin{aligned}
\left|\widetilde{R}^{H} f_{2}(x)\right| & =\left|\int_{\left|x_{0}-z\right|>2 \rho\left(x_{0}\right)} \nabla_{z}^{2} \Gamma(x, z) f(z) d z\right| \\
& \lesssim I_{1}(x)+I_{2}(x),
\end{aligned}
$$

where

$$
\begin{array}{r}
I_{1}(x)=\int_{\left|x_{0}-z\right|>2 \rho\left(x_{0}\right)} \frac{|f(z)|}{(1+|x-z| / \rho(x))^{l}|x-z|^{n}} d z, \\
I_{2}(x)=\int_{\left|x_{0}-z\right|>2 \rho\left(x_{0}\right)} \frac{|f(z)|}{(1+|x-z| / \rho(x))^{l}|x-z|^{n-2}} \\
\quad \times \int_{B(z,|x-z| / 4)} \frac{|\nabla V(u)|}{|u-z|^{n-1}} d u d z .
\end{array}
$$

For $x \in Q$, note that $\rho(x) \sim \rho\left(x_{0}\right)$ by using Lemma 8 . We also note that $|x-z| \sim\left|x_{0}-z\right|$. Then,

$$
\left|I_{1}(x)\right|
$$

$$
\begin{aligned}
& \leq C \sum_{k \geq 1} \frac{2^{-l k}}{\left(2^{k} \rho\left(x_{0}\right)\right)^{n}} \int_{2^{k} \rho\left(x_{0}\right) \leq\left|x_{0}-z\right|<2^{k+1} \rho\left(x_{0}\right)}|f(z)| d z \\
& \leq C \sum_{k \geq 1} \frac{2^{-l k}}{\left(2^{k} \rho\left(x_{0}\right)\right)^{n}} \int_{\left|x_{0}-z\right|<2^{k+1} \rho\left(x_{0}\right)}|f(z)| d z
\end{aligned}
$$$$
\leq C \sum_{k \geq 1} 2^{-l k}\left(\frac{1}{\left|B\left(x_{0}, 2^{k} \rho\left(x_{0}\right)\right)\right|} \int_{\left|x_{0}-z\right|<2^{k+1} \rho\left(x_{0}\right)}|f(z)|^{p} d z\right)^{1 / p}
$$$$
\leq C \inf _{y \in Q} M_{p} f(y) \text {. }
$$ 
Since $x \in Q$, then

$$
\begin{aligned}
& I_{2}(x) \\
& \lesssim \int_{\left|x_{0}-z\right|>2 \rho\left(x_{0}\right)} \frac{|f(z)|}{\left(1+\left|x_{0}-z\right| / \rho\left(x_{0}\right)\right)^{l}\left|x_{0}-z\right|^{n-2}} \\
& \times \int_{B\left(x_{0}, 4\left|x_{0}-z\right|\right)} \frac{|\nabla V(u)|}{|u-z|^{n-1}} d u d z \\
& \lesssim \sum_{k \geq 1} \frac{2^{-l k}}{\left(2^{k} \rho\left(x_{0}\right)\right)^{n-2}} \int_{\left|x_{0}-z\right|<2^{k+1} \rho\left(x_{0}\right)}|f(z)| d z \\
& \times \int_{B\left(x_{0}, 2^{k+3} \rho\left(x_{0}\right)\right)} \frac{|\nabla V(u)|}{|u-z|^{n-1}} \\
& \lesssim \sum_{k \geq 1} \frac{2^{-l k}}{\left(2^{k} \rho\left(x_{0}\right)\right)^{n-2}} \\
& \times \int_{\left|x_{0}-z\right|<2^{k} \rho\left(x_{0}\right)}|f(z)| \mathscr{I}_{1}\left(|\nabla V| \chi_{B\left(x_{0}, 2^{k+3} \rho\left(x_{0}\right)\right)}\right) d z .
\end{aligned}
$$

Using the Hölder inequality and the boundedness of the fractional integral $\mathscr{I}_{1}$ with $1 / s_{1}=1 / p^{\prime}+1 / n$, we have

$$
\begin{aligned}
& \int_{\left|x_{0}-z\right|<2^{k} \rho\left(x_{0}\right)}|f(z)| \mathscr{I}_{1}\left(|\nabla V| \chi_{B\left(x_{0}, 2^{k+3} \rho\left(x_{0}\right)\right)}\right) d z \\
& \quad \times\left\|f \chi_{B\left(x_{0}, 2^{k+3} \rho\left(x_{0}\right)\right)}\right\|\left\|_{p}\right\| \mathscr{I}_{1}\left(|\nabla V| \chi_{B\left(x_{0}, 2^{k+3} \rho\left(x_{0}\right)\right)}\right) \|_{p^{\prime}} \\
& \leq\left\|f \chi_{B\left(x_{0}, 2^{k+3} \rho\left(x_{0}\right)\right)}\right\|_{p}\left\||\nabla V| \chi_{B\left(x_{0}, 2^{k+3} \rho\left(x_{0}\right)\right)}\right\|_{s_{1}} .
\end{aligned}
$$

Since $|\nabla V| \in B_{s_{1}}$, we obtain

$$
\begin{aligned}
& \left\||\nabla V| \chi_{B\left(x_{0}, 2^{k+3} \rho\left(x_{0}\right)\right)}\right\|_{s_{1}} \\
& \quad \lesssim\left(2^{k} \rho\left(x_{0}\right)\right)^{-n / s_{1}^{\prime}} \int_{B\left(x_{0}, 2^{k} \rho\left(x_{0}\right)\right)}|\nabla V(z)| d z \\
& \quad \lesssim\left(2^{k} \rho\left(x_{0}\right)\right)^{n-2-n / s_{1}^{\prime}} \frac{1}{\left(2^{k} \rho\left(x_{0}\right)\right)^{n-2}} \int_{B\left(x_{0}, 2^{k} \rho\left(x_{0}\right)\right)}|\nabla V(z)| d z \\
& \quad \lesssim\left(2^{k} \rho\left(x_{0}\right)\right)^{n-2-n / s_{1}^{\prime}}\left(2^{k} \rho\left(x_{0}\right) m\left(x_{0},|\nabla V|\right)\right)^{k_{0}^{\prime}} \\
& \quad \lesssim\left(2^{k} \rho\left(x_{0}\right)\right)^{n-2-n / s_{1}^{\prime}}\left(2^{k}\right)^{k_{0}^{\prime}},
\end{aligned}
$$

where we use the assumption that $m\left(x_{0},|\nabla V|\right) \lesssim m\left(x_{0}, V\right)$ and (2) in Lemma 10.

We also have

$$
\left\|f \chi_{B\left(x_{0}, 2^{k+3} \rho\left(x_{0}\right)\right)}\right\|_{p} \lesssim\left(2^{k} \rho\left(x_{0}\right)\right)^{n / p} \inf _{y \in Q} M_{p} f(y) \text {. }
$$

Therefore, using the fact that $n / p-n / s_{1}^{\prime}=1$, we obtain

$$
\begin{aligned}
I_{2}(x) \lesssim \sum_{k \geq 1} & \frac{2^{-l k}}{\left(2^{k} \rho\left(x_{0}\right)\right)^{n-2}}\left(2^{k} \rho\left(x_{0}\right)\right)^{n-2-n / s_{1}^{\prime}} \\
& \times\left(2^{k}\right)^{k_{0}^{\prime}}\left(2^{k} \rho\left(x_{0}\right)\right)^{n / p} \inf _{y \in Q} M_{p} f(y)
\end{aligned}
$$

$$
\begin{aligned}
& \lesssim \sum_{k \geq 1} 2^{-l k+k k_{0}^{\prime}+1} \rho\left(x_{0}\right) \inf _{y \in Q} M_{p} f(y) \\
& \lesssim \inf _{y \in Q} M_{p} f(y),
\end{aligned}
$$

where we choose $l$ large enough such that the previous series converges and we use the fact that $\rho\left(x_{0}\right) \lesssim 1$.

To deal with the second term of (45), we split again $f=$ $f_{1}+f_{2}$ with $f_{1}=f \chi_{2 Q}$.

Firstly, using the Hölder inequality and the boundedness of $\widetilde{\mathscr{R}}^{H}$ on $L^{p}\left(\mathbb{R}^{n}\right)$,

$$
\begin{aligned}
& \frac{1}{|Q|} \int_{Q}\left|\widetilde{R}^{H}\left(\left(b-b_{\mathrm{Q}}\right) f_{1}\right)(y)\right| d y \\
& \leq\left(\frac{1}{|Q|} \int_{\mathrm{Q}}\left|\widetilde{\mathscr{R}}^{H}\left(\left(b-b_{\mathrm{Q}}\right) f_{1}\right)(y)\right|^{p_{1}} d y\right)^{1 / p_{1}} \\
& \quad \leq\left(\frac{1}{|Q|} \int_{2 \mathrm{Q}}\left|\left(\left(b-b_{\mathrm{Q}}\right) f\right)(y)\right|^{p_{1}} d y\right)^{1 / p_{1}} \\
& \quad \leq\left(\frac{1}{|Q|} \int_{\mathrm{Q}}\left|b-b_{\mathrm{Q}}\right|^{v} d y\right)^{1 / v}\left(\frac{1}{|Q|} \int_{2 \mathrm{Q}}|f(y)|^{p} d y\right)^{1 / p} \\
& \quad \leq C[b]_{\theta} \inf _{y \in \mathrm{Q}} M_{p} f(y),
\end{aligned}
$$

where $p_{1} / p+p_{1} / v=1, p>p_{1}$, and we have used Lemma 21 in the last inequality.

For the remaining term, we firstly see the fact that $\rho(x) \sim$ $\rho\left(x_{0}\right)$ and $|x-z| \sim\left|x_{0}-z\right|$. Then, we deal with

$$
\begin{aligned}
& \left|\widetilde{\mathscr{R}}^{H}\left[f_{2}\left(b-b_{\mathrm{Q}}\right)\right](x)\right| \\
& \quad=\left|\int_{\left|x_{0}-z\right|>2 \rho\left(x_{0}\right)} \nabla_{z}^{2} \Gamma(x, z)\left[f_{2}\left(b-b_{\mathrm{Q}}\right)\right](z) d z\right| \\
& \quad \leq \widetilde{I}_{1}(x)+\widetilde{I}_{2}(x),
\end{aligned}
$$

where

$$
\begin{aligned}
& \tilde{I}_{1}(x)=\int_{\left|x_{0}-z\right|>2 \rho\left(x_{0}\right)} \frac{\left|f_{2}(z) b-b_{\mathrm{Q}}\right|}{\left|x_{0}-z\right|^{n}\left(1+\left|x_{0}-z\right| / \rho\left(x_{0}\right)\right)^{l}} d z, \\
& \tilde{I}_{2}(x)=\int_{\left|x_{0}-z\right|>2 \rho\left(x_{0}\right)} \frac{\left|f_{2}(z)\left(b-b_{\mathrm{Q}}\right)\right|}{\left(1+\left|x_{0}-z\right| / \rho\left(x_{0}\right)\right)^{l}\left|x_{0}-z\right|^{n-2}} \\
& \quad \times \int_{B\left(z,\left|x_{0}-z\right| / 4\right)} \frac{|\nabla V(u)|}{|u-z|^{n-1}} d u d z .
\end{aligned}
$$

By the Hölder inequality and Lemma 22, we have $\tilde{I}_{1}(x)$

$$
\begin{aligned}
& \lesssim \sum_{k \geq 1} \frac{2^{-l k}}{\left(2^{k} \rho\left(x_{0}\right)\right)^{n}} \int_{2^{k} \rho\left(x_{0}\right) \leq\left|x_{0}-z\right|<2^{k+1} \rho\left(x_{0}\right)}\left|\left[f_{2}\left(b-b_{\mathrm{Q}}\right)\right](z)\right| d z \\
& \leq C \sum_{k \geq 1} \frac{2^{-l k}}{\left(2^{k} \rho\left(x_{0}\right)\right)^{n}} \int_{\left|x_{0}-z\right|<2^{k+1} \rho\left(x_{0}\right)}\left|\left[f_{2}\left(b-b_{\mathrm{Q}}\right)\right](z)\right| d z
\end{aligned}
$$




$$
\begin{aligned}
& \leq C \sum_{k \geq 1} 2^{-l k}\left(\frac{1}{\left|B\left(x_{0}, 2^{k} \rho\left(x_{0}\right)\right)\right|} \int_{\left|x_{0}-z\right|<2^{k+1} \rho\left(x_{0}\right)}|f(z)|^{p} d z\right)^{1 / p} \\
& \quad \times\left(\frac{1}{\left|B\left(x_{0}, 2^{k} \rho\left(x_{0}\right)\right)\right|} \int_{\left|x_{0}-z\right|<2^{k+1} \rho\left(x_{0}\right)}\left|b-b_{\mathrm{Q}}\right|^{p^{\prime}} d z\right)^{1 / p^{\prime}} \\
& \leq C \sum_{k \geq 1} 2^{-l k+\theta^{\prime} k} k[b]_{\theta} \inf _{y \in Q} M_{p} f(y) \\
& \leq C \inf _{y \in Q} M_{p} f(y),
\end{aligned}
$$

where $1 / p+1 / p^{\prime}=1$, and we choose $l$ large enough. The following estimate is similar to the estimate of $I_{2}(x)$. We repeat the previous method.

Then,

$$
\begin{aligned}
& \tilde{I}_{2}(x) \lesssim \int_{\left|x_{0}-z\right|>2 \rho\left(x_{0}\right)} \frac{\left|f_{2}(z)\left(b-b_{\mathrm{Q}}\right)\right|}{\left(1+\left|x_{0}-z\right| / \rho\left(x_{0}\right)\right)^{l}\left|x_{0}-z\right|^{n-2}} \\
& \times \int_{B\left(x_{0}, 4\left|x_{0}-z\right|\right)} \frac{|\nabla V(u)|}{|u-z|^{n-1}} d u d z \\
& \lesssim \sum_{k \geq 1} \frac{2^{-l k}}{\left(2^{k} \rho\left(x_{0}\right)\right)^{n-2}} \\
& \times \int_{\left|x_{0}-z\right|<2^{k+1} \rho\left(x_{0}\right)}\left|f_{2}(z)\left(b-b_{\mathrm{Q}}\right)\right| d z \\
& \times \int_{B\left(x_{0}, 2^{k+3} \rho\left(x_{0}\right)\right)} \frac{|\nabla V(u)|}{|u-z|^{n-1}} \\
& \lesssim \sum_{k \geq 1} \frac{2^{-l k}}{\left(2^{k} \rho\left(x_{0}\right)\right)^{n-2}} \\
& \times \int_{\left|x_{0}-z\right|<2^{k} \rho\left(x_{0}\right)}\left|f_{2}(z)\left(b-b_{\mathrm{Q}}\right)\right| \\
& \times \mathscr{I}_{1}\left(|\nabla V| \chi_{B\left(x_{0}, 2^{k+3} \rho\left(x_{0}\right)\right)}\right) d z .
\end{aligned}
$$

Using the Hölder inequality and the boundedness of the fractional integral $\mathscr{F}_{1}$ with $1 / s_{1}=1 / \tilde{p}^{\prime}+1 / n$, we have

$$
\begin{aligned}
& \int_{\left|x_{0}-z\right|<2^{k} \rho\left(x_{0}\right)}\left|f_{2}(z)\left(b-b_{\mathrm{Q}}\right)\right| \mathscr{I}_{1}\left(|\nabla V| \chi_{B\left(x_{0}, 2^{k+3} \rho\left(x_{0}\right)\right)}\right) d z \\
& \quad \times\left\|f_{2}(z)\left(b-b_{\mathrm{Q}}\right) \chi_{B\left(x_{0}, 2^{k+3} \rho\left(x_{0}\right)\right)}\right\|_{\tilde{p}} \\
& \quad \times\left\|\mathscr{I}_{1}\left(|\nabla V| \chi_{\left.B\left(x_{0}, 2^{k+3} \rho\left(x_{0}\right)\right)\right)}\right)\right\|_{\tilde{p}^{\prime}} \\
& \leq\left\|f_{2}(z)\left(b-b_{\mathrm{Q}}\right) \chi_{B\left(x_{0}, 2^{k+3} \rho\left(x_{0}\right)\right)}\right\|\left\|_{\tilde{p}}\right\||\nabla V| \chi_{B\left(x_{0}, 2^{k+3} \rho\left(x_{0}\right)\right)} \|_{S_{1}} .
\end{aligned}
$$

Since $|\nabla V| \in B_{s_{1}}$, we have already obtained

$$
\left\||\nabla V| \chi_{B\left(x_{0}, 2^{k+3} \rho\left(x_{0}\right)\right)}\right\|_{s_{1}} \leqslant\left(2^{k} \rho\left(x_{0}\right)\right)^{n-2-n / s_{1}^{\prime}}\left(2^{k}\right)^{k_{0}^{\prime}} .
$$

Also,

$$
\begin{aligned}
& \left\|f_{2}(z)\left(b-b_{\mathrm{Q}}\right) \chi_{B\left(x_{0}, 2^{k+3} \rho\left(x_{0}\right)\right)}\right\|_{\tilde{p}} \\
& \leq\left(2^{k} \rho\left(x_{0}\right)\right)^{n / \tilde{p}} \\
& \quad \times\left(\frac{1}{\left|B\left(x_{0}, 2^{k} \rho\left(x_{0}\right)\right)\right|} \int_{\left|x_{0}-z\right|<2^{k} \rho\left(x_{0}\right)}|f(z)|^{p} d z\right)^{1 / p} \\
& \quad \times\left(\frac{1}{\left|B\left(x_{0}, 2^{k} \rho\left(x_{0}\right)\right)\right|} \int_{\left|x_{0}-z\right|<2^{k} \rho\left(x_{0}\right)}\left|b-b_{\mathrm{Q}}\right|^{v} d z\right)^{1 / v} \\
& \leq 2^{k \theta^{\prime}} k\left(2^{k} \rho\left(x_{0}\right)\right)^{n / \tilde{p}}[b]_{\theta} \inf _{y \in \mathrm{Q}} M_{p} f(y),
\end{aligned}
$$

where $\tilde{p} / p+\tilde{p} / \nu=1$.

Therefore, using that $n / p-n / s_{1}^{\prime}=1$, we obtain

$$
\begin{aligned}
& I_{2}(x) \lesssim \sum_{k \geq 1} \frac{2^{-l k} k}{\left(2^{k} \rho\left(x_{0}\right)\right)^{n-2}}\left(2^{k} \rho\left(x_{0}\right)\right)^{n-2-n / s_{1}^{\prime}} \\
& \times\left(2^{k}\right)^{k_{0}^{\prime}+\theta^{\prime}}\left(2^{k} \rho\left(x_{0}\right)\right)^{n / \widetilde{p}}[b]_{\theta} \inf _{y \in Q} M_{p} f(y) \\
& \lesssim \sum_{k \geq 1} k 2^{-l k+k k_{0}^{\prime}+k+k \theta^{\prime}}[b]_{\theta} \rho\left(x_{0}\right) \inf _{y \in Q} M_{p} f(y) \\
& \lesssim[b] \inf _{y \in Q} M_{p} f(y),
\end{aligned}
$$

where we choose $l$ large enough such that the previous series converges and we use the fact that $\rho\left(x_{0}\right) \lesssim 1$.

Therefore, this completes the proof.

Remark 25. Similarly, we can conclude that the previous lemma also holds if the critical ball $Q$ is replaced by $2 Q$.

Lemma 26. Suppose that $V \in B_{s}$ for some $s \geq n,|\nabla V| \in B_{s_{1}}$ $\left(s_{1} \geq n / 2\right), \rho(x) \leqslant \rho_{1}(x)$, and $\rho(x) \lesssim 1$. Let $b \in B M O_{\theta}(\rho)$. Then, there exists a constant $C$ such that

$$
\begin{gathered}
\int_{(2 B)^{c}}\left|\nabla_{z}^{2} \Gamma(x, z)-\nabla_{z}^{2} \Gamma(y, z)\right|\left|b(z)-b_{B}\right||f(z)| d z \\
\leq C[b]_{\theta} \inf _{y \in Q} M_{p} f(y),
\end{gathered}
$$

for all $f \in L_{\text {loc }}^{p}\left(\mathbb{R}^{n}\right)$ for $p>s_{1}^{\prime}$ and $x, y \in B=B\left(x_{0}, r\right)$, with $r<\gamma \rho\left(x_{0}\right)$, where $\gamma \geq 1$.

Proof. Denote that $Q=B\left(x_{0}, \gamma \rho\left(x_{0}\right)\right)$. Note that $\rho(x) \sim \rho\left(x_{0}\right)$ and $|x-z| \sim\left|x_{0}-z\right|$. By the estimate (29), we have

$$
\begin{aligned}
& \int_{(2 B)^{c}}\left|\nabla_{z}^{2} \Gamma(x, z)-\nabla_{z}^{2} \Gamma(y, z)\right|\left|b(z)-b_{B}\right||f(z)| d z \\
& \quad \leqslant r^{\delta} \int_{Q \mid 2 B} \frac{|f(z)|\left|b(z)-b_{B}\right|}{\left|x_{0}-z\right|^{n+\delta}} d z
\end{aligned}
$$




$$
\begin{aligned}
& +r^{\delta} \rho\left(x_{0}\right)^{l} \int_{Q^{c}} \frac{|f(z)|\left|b(z)-b_{B}\right|}{\left|x_{0}-z\right|^{n+\delta+l}} d z \\
& +r^{\delta} \int_{Q \mid 2 B} \frac{\left|f(z)\left(b-b_{Q}\right)\right|}{\left|x_{0}-z\right|^{n-2+\delta}} \int_{B\left(x_{0},\left|x_{0}-z\right| / 4\right)} \frac{|\nabla V(u)|}{|u-z|^{n-1}} d u d z \\
& +r^{\delta} \rho\left(x_{0}\right)^{l} \int_{Q^{c}} \frac{\left|f(z)\left(b-b_{Q}\right)\right|}{\left|x_{0}-z\right|^{n-2+l+\delta}} \\
& \quad \times \int_{B\left(x_{0},\left|x_{0}-z\right| / 4\right)} \frac{|\nabla V(u)|}{|u-z|^{n-1}} d u d z \\
& =I_{1}+I_{2}+I_{3}+I_{4} .
\end{aligned}
$$

For $I_{1}$, by using the Hölder inequality and Lemma 22, we have

$$
\begin{aligned}
I_{1} & \lesssim \sum_{j=2}^{j_{0}} \frac{2^{-j \delta}}{\left|B\left(x_{0}, 2^{j} r\right)\right|} \int_{2^{j} B}|f(z)|\left|b(z)-b_{B}\right| d z \\
& \lesssim \sum_{j=2}^{j_{0}} 2^{-j \delta} j[b]_{\theta}\left(1+\frac{2^{j} r}{\rho\left(x_{0}\right)}\right)^{\theta^{\prime}} \inf _{y \in B} M_{p} f(y) \\
& \lesssim \sum_{j=2}^{\infty} 2^{-j \delta} j[b]_{\theta} \inf _{y \in B} M_{p} f(y) \\
& \lesssim[b]_{\theta} \inf _{y \in B} M_{p} f(y),
\end{aligned}
$$

where $j_{0}$ is the least integer such that $2^{j_{0}} \geq\left(\gamma \rho\left(x_{0}\right)\right) / r$. have

To deal with $I_{2}$, using Lemma 22 and choosing $l>\theta^{\prime}$, we

$$
\begin{aligned}
I_{2} & \lesssim \frac{\rho\left(x_{0}\right)^{l}}{r^{l}} \sum_{j=j_{0}-1}^{\infty} \frac{2^{-j(\delta+l)}}{\left|B\left(x_{0}, 2^{j} r\right)\right|} \int_{2^{j} B}|f(z)|\left|b(z)-b_{B}\right| d z \\
& \lesssim \frac{\rho\left(x_{0}\right)^{l}}{r^{l}} \sum_{j=j_{0}}^{\infty} 2^{-j(\delta+l)} j[b]_{\theta}\left(1+\frac{2^{j} r}{\rho\left(x_{0}\right)}\right)^{\theta^{\prime}} \inf _{y \in B} M_{p} f(y) \\
& \lesssim \sum_{j=j_{0}}^{\infty} j 2^{-j \delta}\left(\frac{\rho\left(x_{0}\right)}{2^{j} r}\right)^{l-\theta^{\prime}}[b]_{\theta} \inf _{y \in B} M_{p} f(y) \\
& \lesssim[b]_{\theta} \inf M_{y \in B} f(y),
\end{aligned}
$$

where we use the fact that $\rho\left(x_{0}\right) / 2^{j} r \leq 1 / \gamma$ when $j>j_{0}$.

To deal with $I_{3}$, by using Lemma 22 and $j \leq j_{0}$,

$$
\begin{gathered}
I_{3} \lesssim \frac{1}{r^{n-2}} \sum_{j=2}^{j_{0}} 2^{-j(n-2+\delta)} \\
\quad \times \int_{2^{j} B}|f(z)|\left|b(z)-b_{B}\right| \mathscr{I}_{1}\left(\nabla V \chi_{2^{j+2} B}\right)(z) d z \\
\leq \frac{1}{r^{n-2}} \sum_{j=2}^{j_{0}} 2^{-j(n-2+\delta)}\left\|f \chi_{2^{j} B}\right\|_{p} \\
\quad \times\left\|\left(b-b_{B}\right) \chi_{2^{j} B}\right\|\left\|_{\nu}\right\| \mathscr{I}_{1}\left(\nabla V \chi_{2^{j+2} B}\right) \|_{\tilde{p}^{\prime}}
\end{gathered}
$$

$$
\begin{aligned}
\lesssim \frac{1}{r^{n-2}} \sum_{j=2}^{j_{0}} 2^{-j(n-2+\delta)} j\left|2^{j} B\right|^{1 / \widetilde{p}} \\
\quad \times[b]_{\theta} \inf _{y \in B} M_{p} f(y)\left\|\nabla V \chi_{2^{j+2} B}\right\|_{s_{1}}
\end{aligned}
$$

where $1 / s_{1}=1 / \tilde{p}^{\prime}+1 / n$ and $1 / p+1 / \nu+1 / \tilde{p}^{\prime}=1$.

Since $|\nabla V| \in B_{s_{1}}$, then

$$
\begin{aligned}
\left\|\nabla V \chi_{2^{j+2} B}\right\|_{s_{1}} & \lesssim\left\|\nabla V \chi_{\mathrm{Q}}\right\|_{s_{1}} \\
& \lesssim \rho\left(x_{0}\right)^{-n / s_{1}^{\prime}} \int_{Q}|\nabla V(z)| d z \\
& \lesssim \rho\left(x_{0}\right)^{n / s_{1}-2},
\end{aligned}
$$

for all $j \leq j_{0}$, where we use the fact that $m\left(x_{0},|\nabla V|\right) \lesssim$ $m\left(x_{0}, V\right)$.

Therefore,

$$
\begin{aligned}
I_{3} & \leqslant[b]_{\theta} \inf _{y \in B} M_{p} f(y) \frac{r^{n / \tilde{p}-n+2}}{\rho\left(x_{0}\right)^{2-n / s_{1}}} \sum_{j=2}^{j_{0}} j 2^{-j(n-2+\delta-n / \tilde{p})} \\
& \lesssim[b]_{\theta} \inf _{y \in B} M_{p} f(y) r\left(\frac{r}{\rho\left(x_{0}\right)}\right)^{2-n / s_{1}} \sum_{j=2}^{j_{0}} j 2^{-j(n /-3+\delta)} \\
& \lesssim[b]_{\theta} \inf _{y \in B} M_{p} f(y)\left(\frac{r}{\rho\left(x_{0}\right)}\right)^{2-n / s_{1}} 2^{j_{0}\left(3-n / s_{1}\right)} \sum_{j=2}^{j_{0}} j 2^{-j \delta} \\
& \lesssim[b]_{\theta} \inf _{y \in B} M_{p} f(y),
\end{aligned}
$$

where we use that $r \leq \gamma \rho\left(x_{0}\right) \lesssim 1$.

At last, for $I_{4}$ we have, for $j>j_{0}$,

$I_{4}$

$$
\begin{aligned}
& \lesssim \frac{\rho\left(x_{0}\right)^{l}}{r^{n-2+l}} \sum_{j=j_{0}-1}^{\infty} 2^{-j(n-2+\delta+l)} \\
& \quad \times \int_{2^{j} B}|f(z)|\left|b(z)-b_{B}\right| \mathscr{I}_{1}\left(\nabla V \chi_{2^{j+2} B}\right)(z) d z
\end{aligned}
$$

$$
\begin{gathered}
\lesssim \frac{\rho\left(x_{0}\right)^{l}}{r^{n-2+l}} \sum_{j=j_{0}-1}^{\infty} 2^{-j(n-2+\delta+l)}\left\|f \chi_{2^{j} B}\right\|_{p} \\
\quad \times\left\|\left(b-b_{B}\right) \chi_{2^{j} B}\right\|_{\nu}\left\|\mathscr{I}_{1}\left(\nabla V \chi_{2^{j+2} B}\right)\right\|_{\tilde{p}^{\prime}} \\
\leq \frac{\rho\left(x_{0}\right)^{l}}{r^{n-2+l}} \sum_{j=j_{0}-1}^{\infty} 2^{-j(n-2+\delta+l)} j \frac{\left(2^{j} r\right)^{\theta^{\prime}+n / \tilde{p}}}{\rho\left(x_{0}\right)^{\theta^{\prime}}} \\
\quad \times[b]_{\theta} \inf _{y \in B} M_{p} f(y)\left\|\nabla V \chi_{2^{j+2} B}\right\|_{s_{1}},
\end{gathered}
$$

where $1 / s_{1}=1 / \widetilde{p}^{\prime}+1 / n$ and $1 / p+1 / \nu+1 / \tilde{p}^{\prime}=1$. 
Furthermore, by using Lemma 7,

$$
\begin{aligned}
\left\|\nabla V \chi_{2^{j+2} B}\right\|_{s_{1}} & \lesssim\left(2^{j} r\right)^{-n / s_{1}^{\prime}} \int_{2^{j} B}|\nabla V(z)| d z \\
& =\left(2^{j} r\right)^{-n / s_{1}^{\prime}} \int_{\left.B\left(x_{0}, 2^{j} r / \rho\left(x_{0}\right)\right) \rho\left(x_{0}\right)\right)}|\nabla V(z)| d z \\
& \lesssim\left(2^{j} r\right)^{n \mu-n / s_{1}^{\prime}} \frac{1}{\rho\left(x_{0}\right)^{n \mu}} \int_{Q}|\nabla V(z)| d z \\
& \lesssim\left(2^{j} r\right)^{n \mu-n / s_{1}^{\prime}} \frac{1}{\rho\left(x_{0}\right)^{n \mu}} \frac{\rho\left(x_{0}\right)^{n-2}}{\rho\left(x_{0}\right)^{n-2}} \int_{Q}|\nabla V(z)| d z \\
& \lesssim\left(2^{j} r\right)^{n \mu-n / s_{1}^{\prime}} \frac{\rho\left(x_{0}\right)^{n-2}}{\rho\left(x_{0}\right)^{n \mu}}\left(1+\rho\left(x_{0}\right) m\left(x_{0},|\nabla V|\right)\right)^{k_{0}^{\prime}} \\
& \lesssim\left(2^{j} r\right)^{n \mu-n / s_{1}^{\prime}} \frac{\rho\left(x_{0}\right)^{n-2}}{\rho\left(x_{0}\right)^{n \mu}},
\end{aligned}
$$

where we use the fact that $m\left(x_{0},|\nabla V|\right) \lesssim m\left(x_{0}, V\right)$. Consider

$$
\begin{aligned}
& I_{4} \lesssim \frac{\rho\left(x_{0}\right)^{l}}{r^{n-2+l}} \sum_{j=j_{0}-1}^{\infty} 2^{-j(n-2+\delta+l)} j \frac{\left(2^{j} r\right)^{\theta^{\prime}+n / \tilde{p}}}{\rho\left(x_{0}\right)^{\theta^{\prime}}} \\
& \times[b]_{\theta} \inf _{y \in B} M_{p} f(y)\left(2^{j} r\right)^{n \mu-n / s_{1}^{\prime}} \frac{\rho\left(x_{0}\right)^{n-2}}{\rho\left(x_{0}\right)^{n \mu}} \\
& \lesssim \frac{\rho\left(x_{0}\right)^{l}}{r^{n-2+l}} \sum_{j=j_{0}-1}^{\infty} 2^{-j(n-2+\delta+l)} j \frac{\left(2^{j} r\right)^{\theta^{\prime}+1}}{\rho\left(x_{0}\right)^{\theta^{\prime}}} \\
& \times[b]_{\theta} \inf _{y \in B} M_{p} f(y)\left(2^{j} r\right)^{n \mu} \frac{\rho\left(x_{0}\right)^{n-2}}{\rho\left(x_{0}\right)^{n \mu}} \\
& \lesssim \frac{\rho\left(x_{0}\right)^{l}}{r^{n-2+l}} \sum_{j=j_{0}-1}^{\infty} 2^{-j(n-2+\delta+l)} j \frac{\left(2^{j} r\right)^{\theta^{\prime}+1}}{\rho\left(x_{0}\right)^{\theta^{\prime}}} \\
& \times[b]_{\theta} \inf _{y \in B} M_{p} f(y)\left(2^{j} r\right)^{n \mu} \frac{\rho\left(x_{0}\right)^{n-2}}{\rho\left(x_{0}\right)^{n \mu}} \\
& \lesssim[b]_{\theta} \inf _{y \in B} M_{p} f(y)\left(\frac{\rho\left(x_{0}\right)}{r}\right)^{n-2+l-\theta^{\prime}-1-n \mu} \\
& \times \rho\left(x_{0}\right) \sum_{j=j_{0}-1}^{\infty} 2^{-j\left(\delta+n-2+l-n \mu-\theta^{\prime}-1\right)} j \\
& \lesssim[b]_{\theta} \inf _{y \in B} M_{p} f(y),
\end{aligned}
$$

where we choose $l$ large enough such that the previous series converges and we use the fact that $\rho\left(x_{0}\right) \lesssim 1$.

Proof of Theorem 4. We start with a function $f \in L^{p}\left(\mathbb{R}^{n}\right)$ for $s_{1}^{\prime}<p<\infty$. By Lemmas 24 and 26 and Remark 25, we have

$$
\begin{aligned}
\left\|\widetilde{\mathscr{R}}_{b}^{H} f\right\|_{L^{q}}^{q} \leq & \int_{\mathbb{R}^{n}}\left|M_{\rho, \beta}\left(\widetilde{\mathscr{R}}_{b}^{H} f\right)(x)\right|^{q} d x \\
\leq & \int_{\mathbb{R}^{n}}\left|M_{\rho, \gamma}^{\sharp}\left(\widetilde{\mathscr{R}}_{b}^{H} f\right)(x)\right|^{q} d x \\
& +C \sum_{k}\left|Q_{k}\right|\left(\frac{1}{\left|Q_{k}\right|} \int_{2 Q_{k}}\left|\widetilde{\mathscr{R}}_{b}^{H} f(x)\right| d x\right)^{q} \\
\leq & C \int_{\mathbb{R}^{n}}\left|M_{\rho, \gamma}^{\sharp}\left(\widetilde{\mathscr{R}}_{b}^{H} f\right)(x)\right|^{q} d x \\
& +C[b]_{\theta}^{p} \sum_{k} \int_{2 Q_{k}}\left|M_{p} f(x)\right|^{q} d x \\
\leq & C \int_{\mathbb{R}^{n}}\left|M_{\rho, \gamma}^{\sharp}\left(\widetilde{\mathscr{R}}_{b}^{H} f\right)(x)\right|^{q} d x+C[b]_{\theta}^{q}\|f\|_{L^{q}}^{q},
\end{aligned}
$$

where we use the finite overlapping property given by Proposition 20 and the boundedness of $M_{p}$ in $L^{q}\left(\mathbb{R}^{n}\right)$ for $p<q$.

Next, we consider the term $\int_{\mathbb{R}^{n}}\left|M_{\rho, \gamma}^{\sharp}\left(\widetilde{\mathscr{R}}_{b}^{H} f\right)(x)\right|^{q} d x$. Our goal is to find a pointwise estimate of $M_{\rho, \gamma}^{\sharp}\left(\widetilde{\mathscr{R}}_{b}^{H} f\right)(x)$. Let $x \in$ $\mathbb{R}^{n}$ and $B=B\left(x_{0}, r\right)$, with $r<\gamma \rho\left(x_{0}\right)$ such that $x \in B$. If $f=f_{1}+f_{2}$, with $f_{1}=f \chi_{2 B}$, then we write

$$
\begin{aligned}
\widetilde{\mathscr{R}}_{b}^{H} f= & \left(b-b_{B}\right) \widetilde{\mathscr{R}}^{H} f-\widetilde{\mathscr{R}}^{H}\left(f_{1}\left(b-b_{B}\right)\right) \\
& -\widetilde{\mathscr{R}}^{H}\left(f_{2}\left(b-b_{B}\right)\right) .
\end{aligned}
$$

Therefore, we need to control the mean oscillation on $B$ of each term that we call $J_{1}, J_{2}$, and $J_{3}$. By using the Hölder inequality and Lemma 21, we obtain

$$
\begin{aligned}
J_{1} & \leq \frac{2}{|B|} \int_{B}\left|\left(b-b_{B}\right) \widetilde{\mathscr{R}}^{H} f(x)\right| d x \\
& \leq\left(\frac{2}{|B|} \int_{B}\left|b-b_{B}\right|^{p^{\prime}} d x\right)^{1 / p^{\prime}}\left(\frac{1}{|B|} \int_{B}\left|\widetilde{\mathscr{R}}^{H} f(x)\right|^{p} d x\right)^{1 / p} \\
& \leq C[b]_{\theta} M_{p}\left(\widetilde{\mathscr{R}}^{H} f\right)(x),
\end{aligned}
$$

since $r / \rho\left(x_{0}\right)<\gamma$.

To estimate $J_{2}$, let $1<\widetilde{p}<p$. Then,

$$
\begin{aligned}
J_{2} & \leq \frac{2}{|B|} \int_{B}\left|\widetilde{\mathscr{R}}^{H}\left[\left(b-b_{B}\right) f_{1}\right](x)\right| d x \\
& \leq\left(\frac{1}{|B|} \int_{B}\left|\widetilde{\mathscr{R}}^{H}\left[\left(b-b_{B}\right) f_{1}\right](x)\right|^{\widetilde{p}} d x\right)^{1 / \widetilde{p}} \\
& \lesssim\left(\frac{1}{|B|} \in \mathscr{R}_{b}^{H}\left|\left(b-b_{B}\right) f_{1}(x)\right|^{\widetilde{s}} d x\right)^{1 / \widetilde{s}} \\
& \lesssim\left(\frac{1}{|B|} \int_{2 B}\left|b-b_{B}\right|^{v} d x\right)^{1 / v}\left(\frac{1}{|B|} \int_{2 B}|f(x)|^{p} d x\right)^{1 / p} \\
& \lesssim[b]_{\theta} M_{p}(f)(x),
\end{aligned}
$$

where $v=p \widetilde{p} /(p-\tilde{p})$. 
For $J_{3}$, by Lemma 26 , we obtain

$$
\begin{gathered}
J_{3} \leq C \frac{1}{|B|^{2}} \iint_{B} \mid \widetilde{\mathscr{R}}^{H}\left(f_{2}\left(b-b_{B}\right)\right)(u) \\
-\left(\widetilde{\mathscr{R}}^{H} f_{2}\left(b-b_{B}\right)\right)(y) \mid d u d y \\
\leq C \frac{1}{|B|^{2}} \iint_{B} \int_{(2 B)^{c}}\left|\nabla^{2} \Gamma(u, z)-\nabla^{2} \Gamma(y, z)\right| \\
\quad \times\left|b(z)-b_{B}\right||f(z)| d z d u d y \\
\leq C[b]_{\theta} M_{p} f(x) .
\end{gathered}
$$

Therefore, we have proved that

$$
\left|M_{\rho, \gamma}^{\sharp}\left(\widetilde{\mathscr{R}}_{b}^{H} f\right)(x)\right| \leq C[b]_{\theta}\left(M_{p} \widetilde{\mathscr{R}}^{H} f(x)+M_{p} f(x)\right) .
$$

Then, we have obtained the desired result.

Proof of Theorem 6. For $f \in H_{L}^{1}\left(\mathbb{R}^{n}\right)$, we can write $f=$ $\sum_{j=-\infty}^{\infty} \lambda_{j} a_{j}$, where each $a_{j}$ is a $(1, q)_{\rho}$ atom and $\sum_{j=-\infty}^{\infty}\left|\lambda_{j}\right| \leq$ $2\|f\|_{H_{L}^{1}}$. Suppose that supp $a_{j} \subseteq B_{j}=B\left(x_{j}, r_{j}\right)$ with $r_{j}<$ $\rho\left(x_{j}\right)$. Write

$$
\begin{aligned}
\mathscr{R}_{b}^{H} f(x)= & \sum_{j=-\infty}^{\infty} \lambda_{j}\left(b(x)-b_{B_{j}}\right) \mathscr{R}^{H} a_{j}(x) \chi_{8 B_{j}}(x) \\
& +\sum_{j: r_{j} \geq \rho\left(x_{j}\right) / 4} \lambda_{j}\left(b(x)-b_{B_{j}}\right) \mathscr{R}^{H} a_{j}(x) \chi_{\left(8 B_{j}\right)^{c}}(x) \\
& +\sum_{j: r_{j}<\rho\left(x_{j}\right) / 4} \lambda_{j}\left(b(x)-b_{B_{j}}\right) \mathscr{R}^{H} a_{j}(x) \chi_{\left(8 B_{j}\right)^{c}}(x) \\
& -\mathscr{R}^{H}\left(\sum_{j=-\infty}^{\infty} \lambda_{j}\left(b-b_{B_{j}}\right) a_{j}\right)(x) \\
= & A_{1}(x)+A_{2}(x)+A_{3}(x)+A_{4}(x) .
\end{aligned}
$$

Using the Hölder inequality, the $\left(L^{q}, L^{q}\right)$ boundedness of $\mathscr{R}^{H}$ with $1<q<s$, and Lemma 21 ,

$$
\begin{aligned}
& \left\|\left(b(x)-b_{B}\right) \mathscr{R}^{H} a_{j}(x) \chi_{8 B_{j}}(x)\right\|_{L^{1}\left(\mathbb{R}^{n}\right)} \\
& \leq\left(\int_{8 B_{j}}\left|b(x)-b_{B}\right|^{q^{\prime}} d x\right)^{1 / q^{\prime}}\left\|\mathscr{R}^{H} a_{j}\right\|_{L^{q}} \\
& \leq\left(\int_{8 B_{j}}\left|b(x)-b_{B}\right|^{q^{\prime}} d x\right)^{1 / q^{\prime}}\left\|a_{j}\right\|_{L^{q}} \\
& \leq\left(\frac{1}{\left|B_{j}\right|} \int_{8 B_{j}}\left|b(x)-b_{B}\right|^{q^{\prime}} d x\right)^{1 / q^{\prime}} \\
& \quad \leq C[b]_{\theta},
\end{aligned}
$$

since $r_{j}<\rho\left(x_{j}\right)$.
When we consider the term $A_{2}(x)$, we note that $\rho\left(x_{j}\right)>$ $r_{j} \geq \rho\left(x_{j}\right) / 4$. Consider

$$
\begin{aligned}
& \left\|\left(b(x)-b_{B_{j}}\right) \mathscr{R}^{H} a_{j}(x) \chi_{\left(8 B_{j}\right)^{c}}(x)\right\|_{L^{1}\left(\mathbb{R}^{n}\right)} \\
& \leq \int_{B_{j}}\left|a_{j}(y)\right| d y\left\{\int_{\left|x-x_{j}\right| \geq 8 r_{j}}\left|\nabla^{2} \Gamma(x, y)\right|\left|b(x)-b_{B_{j}}\right| d x\right\} \\
& \leq \int_{B_{j}}\left|a_{j}(y)\right| d y \\
& \quad \times\left\{\int_{\left|x-x_{j}\right| \geq 8 r_{j}} \frac{C_{l}}{(1+|x-y| / \rho(x))^{l}} \frac{1}{|x-y|^{n-2}}\right. \\
& \quad \times\left(\int_{B(x, 2|x-y|)} \frac{|\nabla V(z)|}{|x-z|^{n-1}} d z+\frac{1}{|x-y|^{2}}\right) \\
& \left.\quad \times\left|b(x)-b_{B_{j}}\right| d x\right\} \\
& \leq \int_{B_{j}}\left|a_{j}(y)\right| d y\left\{I_{1}+I_{2}\right\} .
\end{aligned}
$$

Note that $\left|x-x_{j}\right| \sim|x-y|$ and

$$
\left(1+\frac{|x-y|}{\rho(x)}\right) \geq C\left(1+\frac{\left|x-x_{j}\right|}{\rho(x)}\right) \geq C\left(1+\frac{\left|x-x_{j}\right|}{\rho\left(x_{j}\right)}\right)^{1 /\left(k_{0}+1\right)} \text {. }
$$

Then, by Lemma 22,

$$
\begin{aligned}
I_{2} \leqslant \sum_{k=1}^{\infty} \int_{2^{k+3} r_{j} \leq\left|x-x_{j}\right|<2^{k+4} r_{j}} & \frac{1}{\left(1+\left|x-x_{j}\right| \rho\left(x_{j}\right)^{-1}\right)^{l /\left(k_{0}+1\right)}} \\
& \times \frac{1}{\left|x-x_{j}\right|^{n}}\left|b(x)-b_{B_{j}}\right| d x
\end{aligned}
$$

$$
\begin{aligned}
& \lesssim \sum_{k=1}^{\infty} 2^{-(k+1) l /\left(k_{0}+1\right)} \frac{1}{\left|B\left(x, 2^{k+3} r_{j}\right)\right|} \\
& \times \int_{\left|x-x_{j}\right|<2^{k+4} r_{j}}\left|b-b_{B_{j}}\right| d x \\
& \lesssim \sum_{k=1}^{\infty} 2^{-(k+1) l /\left(k_{0}+1\right)} \frac{1}{\left|B\left(x, 2^{k+4} r_{j}\right)\right|} \\
& \times \int_{\left|x-x_{j}\right|<2^{k+4} r_{j}}\left|b-b_{B_{j}}\right| d x \\
& \lesssim \sum_{k=1}^{\infty} 2^{-(k+1) l /\left(k_{0}+1\right)}[b]_{\theta} k\left(1+\frac{2^{k+4} r_{j}}{\rho\left(x_{j}\right)}\right)^{\left(k_{0}+1\right) \theta} \\
& \leq \mathrm{C}[b]_{\theta} \text {, }
\end{aligned}
$$

where we choose $l$ large enough. 
Similarly,

$I_{1}$

$$
\begin{aligned}
\lesssim \sum_{k=1}^{\infty} \int_{2^{k+3} r_{j} \leq\left|x-x_{j}\right|<2^{k+4} r_{j}} & \frac{1}{\left(1+\left|x-x_{j}\right| \rho\left(x_{j}\right)^{-1}\right)^{l /\left(k_{0}+1\right)}} \\
& \times \frac{1}{\left|x-x_{j}\right|^{n-2}} \mathscr{J}_{1}\left(\nabla V \chi_{2^{k+4} B_{j}}\right) \\
& \times(x)\left|b(x)-b_{B_{j}}\right| d x
\end{aligned}
$$

$\leqslant \sum_{k=1}^{\infty} 2^{-(k+1) l /\left(k_{0}+1\right)} \frac{1}{\left(2^{k+3} r_{j}\right)^{n-2}}$

$$
\times \int_{\left|x-x_{j}\right|<2^{k+4} r_{j}} \mathscr{I}_{1}\left(\nabla V \chi_{2^{k+4} B_{j}}\right)(x)\left|b-b_{B_{j}}\right| d x
$$

$\lesssim \sum_{k=1}^{\infty} 2^{-(k+1) l /\left(k_{0}+1\right)}\left(2^{k+3} r_{j}\right)^{2}$

$$
\begin{aligned}
& \times\left(\frac{1}{\left(2^{k+3} r_{j}\right)^{n}} \int_{\left|x-x_{j}\right| 2^{k+4} r_{j}}\left|b-b_{B_{j}}\right|^{q} d x\right)^{1 / q} \\
& \times\left(2^{k+3} r_{j}\right)^{-n / q^{\prime}}\left\|\mathcal{I}_{1}\left(\nabla V \chi_{2^{k+4} B_{j}}\right)\right\|_{q^{\prime}}
\end{aligned}
$$

$\lesssim \sum_{k=1}^{\infty} 2^{-(k+1) l /\left(k_{0}+1\right)}\left(2^{k+3} r_{j}\right)^{2}$

$$
\begin{aligned}
& \times\left(\frac{1}{\left(2^{k+3} r_{j}\right)^{n}} \int_{\left|x-x_{j}\right|<2^{k+4} r_{j}}\left|b-b_{B_{j}}\right|^{q} d x\right)^{1 / q} \\
& \times\left(2^{k+3} r_{j}\right)^{-n / q^{\prime}}\left\|\nabla V \chi_{2^{k+4} B_{j}}\right\|_{s_{1}} \\
& \leq \sum_{k=1}^{\infty} 2^{-(k+1) l\left(k_{0}+1\right)}[b]_{\theta} k\left(1+\frac{2^{k+4} r_{j}}{\rho\left(x_{j}\right)}\right)^{\left(k_{0}+1\right) \theta} \\
& \quad \times\left(2^{k} \rho\left(x_{j}\right)\right)^{n-n / s_{1}^{\prime}-n / q^{\prime}}\left(2^{k}\right)^{k_{0}^{\prime}} \\
& \leq \sum_{k=1}^{\infty} 2^{-(k+1) l\left(k_{0}+1\right)}[b]_{\theta} k\left(1+\frac{2^{k+4} r_{j}}{\rho\left(x_{j}\right)}\right)^{\left(k_{0}+1\right) \theta} \\
& \quad \times\left(2^{k} \rho\left(x_{j}\right)\right)\left(2^{k}\right)^{k_{0}^{\prime}}
\end{aligned}
$$$$
\leq C[b]_{\theta},
$$

where we choose $l$ large enough and we use the fact that $\rho\left(x_{j}\right) \lesssim 1$.

Therefore, if $\rho\left(x_{j}\right)>r_{j} \geq \rho\left(x_{j}\right) / 4$, then

$$
\left\|\left(b(x)-b_{B_{j}}\right) \mathscr{R}^{H} a_{j}(x) \chi_{\left(8 B_{j}\right)^{c}}(x)\right\|_{L^{1}\left(\mathbb{R}^{n}\right)} \leq C[b]_{\theta} .
$$

For $A_{3}$, by using the vanishing condition of $a_{j}$ and Lemma 14, then

$$
\begin{aligned}
& \left\|\left(b(x)-b_{B_{j}}\right) \mathscr{R}^{H} a_{j}(x) \chi_{\left(8 B_{j}\right)^{c}}(x)\right\|_{L^{1}\left(\mathbb{R}^{n}\right)} \\
& \lesssim \int_{B_{j}}\left|a_{j}(y)\right| d y\left\{\int_{\left|x-x_{j}\right| \geq 8 r_{j}}\left|\nabla^{2} \Gamma(x, y)-\nabla^{2} \Gamma\left(x, x_{j}\right)\right|\right. \\
& \left.\times\left|b(x)-b_{B_{j}}\right| d x\right\} \\
& \lesssim \int_{B_{j}}\left|a_{j}(y)\right| d y \\
& \times\left\{\int_{\left|x-x_{j}\right| \geq 8 r_{j}} \frac{C_{l}}{(1+|x-y| / \rho(x))^{l}} \frac{\left|y-x_{j}\right|^{\delta}}{|x-y|^{n-2+\delta}}\right. \\
& \times\left(\int_{B(x, 2|x-y|)} \frac{|\nabla V(z)|}{|x-z|^{n-1}} d z+\frac{1}{|x-y|^{2}}\right) \\
& \left.\times\left|b(x)-b_{B_{j}}\right| d x\right\} \\
& =\int_{B_{j}}\left|a_{j}(y)\right| d y\left\{\widetilde{I}_{1}+\widetilde{I}_{2}\right\} .
\end{aligned}
$$

First of all, we need to obtain the following new estimate:

$$
\begin{aligned}
& \left\||\nabla V| \chi_{B\left(x_{j}, 2^{k+3} r_{j}\right)}\right\|_{s_{1}} \\
& \quad \leq\left(2^{k} r_{j}\right)^{-n / s_{1}^{\prime}} \int_{B\left(x_{j}, 2^{k} r_{j}\right)}|\nabla V(z)| d z \\
& \quad \leq\left(2^{k} r_{j}\right)^{n-2-n / s_{1}^{\prime}} \frac{1}{\left(2^{k} r_{j}\right)^{n-2}} \int_{B\left(x_{j}, 2^{k} r_{j}\right)}|\nabla V(z)| d z \\
& \leq\left(2^{k} r_{j}\right)^{n-2-n / s_{1}^{\prime}}\left(1+2^{k} r_{j} m\left(x_{j},|\nabla V|\right)\right)^{k_{0}^{\prime}} \\
& \leq\left(2^{k} r_{j}\right)^{n-2-n / s_{1}^{\prime}}\left(1+2^{k} r_{j} m\left(x_{j}, V\right)\right)^{k_{0}^{\prime}},
\end{aligned}
$$

where we use the assumption that $m\left(x_{0},|\nabla V|\right) \lesssim m\left(x_{0}, V\right)$ and (2) in Lemma 10. Consider

$\widetilde{I}_{1}$

$\lesssim \int_{B_{j}}\left|a_{j}(y)\right| d y$

$$
\begin{aligned}
& \times \sum_{k=1}^{\infty} \int_{2^{k+3} r_{j} \leq\left|x-x_{j}\right|<2^{k+4} r_{j}} \frac{\mathscr{I}_{1}\left(\nabla V \chi_{2^{k+4} B_{j}}\right)(x)}{\left(1+\left|x-x_{j}\right| \rho\left(x_{j}\right)^{-1}\right)^{l /\left(l_{0}+1\right)}} \\
& \times \frac{\left|x_{j}-y\right|^{\delta}\left|b(x)-b_{B_{j}}\right|}{\left|x-x_{j}\right|^{n-2}\left|x-x_{j}\right|^{\delta}} d x
\end{aligned}
$$




$$
\begin{aligned}
& \lesssim \sum_{k=1}^{\infty} 2^{-(k+3) \delta} \frac{\left(2^{k+3} r_{j}\right)^{2-n / q^{\prime}}}{\left(1+2^{k} r_{j} \rho\left(x_{j}\right)^{-1}\right)^{l /\left(l_{0}+1\right)}} \\
& \times\left(\frac{1}{\left|B\left(x, 2^{k+3} r_{j}\right)\right|} \int_{\left|x-x_{j}\right|<2^{k+4} r_{j}}\left|b-b_{B_{j}}\right|^{q} d x\right)^{1 / q} \\
& \times\left\|\mathscr{I}_{1}\left(\nabla V \chi_{2^{k+4} B_{j}}\right)\right\|_{q^{\prime}} \\
& \lesssim \sum_{k=1}^{\infty} 2^{-(k+3) \delta} \frac{\left(2^{k+3} r_{j}\right)^{2-n / q^{\prime}}}{\left(1+2^{k} r_{j} \rho\left(x_{j}\right)^{-1}\right)^{l /\left(l_{0}+1\right)}} \\
& \times\left(\frac{1}{\left|B\left(x, 2^{k+3} r_{j}\right)\right|} \int_{\left|x-x_{j}\right|<2^{k+4} r_{j}}\left|b-b_{B_{j}}\right|^{q} d x\right)^{1 / q} \\
& \times\left\|\nabla V \chi_{2^{k+4} B_{j}}\right\|_{s_{1}} \\
& \lesssim \sum_{k=1}^{\infty} 2^{-(k+3) \delta}[b]_{\theta} k\left(1+\frac{2^{k+4} r_{j}}{\rho\left(x_{j}\right)}\right)^{\left(k_{0}+1\right) \theta} \\
& \times\left(2^{k} r_{j}\right)^{n-2-n / s_{1}^{\prime}+2-n / q^{\prime}}\left(1+2^{k} r_{j} m\left(x_{j}, V\right)\right)^{k_{0}^{\prime}-l /\left(l_{0}+1\right)} \\
& \lesssim \sum_{k=1}^{\infty} 2^{-(k+3) \delta+k\left(k_{0}+1\right) \theta}[b]_{\theta} k\left(2^{k} r_{j}\right) \\
& \times\left(1+2^{k} r_{j} m\left(x_{j}, V\right)\right)^{k_{0}^{\prime}-l /\left(l_{0}+1\right)+1}\left(1+2^{k} r_{j} m\left(x_{j}, V\right)\right)^{-1} \\
& \lesssim \sum_{k=1}^{\infty} 2^{-(k+3) \delta+k\left(k_{0}+1\right) \theta}[b]_{\theta} k\left(2^{k} r_{j}\right)\left(2^{k} r_{j} m\left(x_{j}, V\right)\right)^{-1} \\
& =\sum_{k=1}^{\infty} 2^{-(k+3) \delta+k\left(k_{0}+1\right) \theta}[b]_{\theta} k \rho\left(x_{j}\right) \\
& \leqslant[b]_{\theta},
\end{aligned}
$$

where we use the fact that $\delta>\left(k_{0}+1\right) \theta, \rho\left(x_{j}\right) \lesssim 1$, and we choose $l>\left(k_{0}^{\prime}+1\right) /\left(l_{0}+1\right)$.

Secondly,

$\widetilde{I}_{2}$

$$
\begin{aligned}
\leq \int_{B_{j}}\left|a_{j}(y)\right| d y & \\
\times \sum_{k=1}^{\infty} \int_{2^{k+3} r_{j} \leq\left|x-x_{j}\right|<2^{k+4} r_{j}} & \frac{C_{l}}{\left(1+\left|x-x_{j}\right| \rho\left(x_{j}\right)^{-1}\right)^{l /\left(l_{0}+1\right)}} \\
& \times \frac{\left|x_{j}-y\right|^{\delta}\left|b(x)-b_{B_{j}}\right|}{\left|x-x_{j}\right|^{n}\left|x-x_{j}\right|^{\delta}} d x
\end{aligned}
$$

$$
\begin{aligned}
& \lesssim \sum_{k=1}^{\infty} 2^{-(k+3) \delta} \frac{1}{\left|B\left(x, 2^{k+3} r_{j}\right)\right|} \int_{\left|x-x_{j}\right|<2^{k+4} r_{j}}\left|b-b_{B_{j}}\right| d x \\
& \leq \sum_{k=1}^{\infty} 2^{-(k+3) \delta} \frac{1}{\left|B\left(x, 2^{k+4} r_{j}\right)\right|} \int_{\left|x-x_{j}\right|<2^{k+4} r_{j}}\left|b-b_{B_{j}}\right| d x \\
& \leq \sum_{k=1}^{\infty} 2^{-(k+3) \delta}[b]_{\theta} k\left(1+\frac{2^{k+4} r_{j}}{\rho\left(x_{j}\right)}\right)^{\left(k_{0}+1\right) \theta} \\
& \lesssim \sum_{k=1}^{\infty} 2^{-(k+3) \delta+k\left(k_{0}+1\right) \theta}[b]_{\theta} k \\
& \leq[b]_{\theta}
\end{aligned}
$$

where we use the fact that $\delta>\left(k_{0}+1\right) \theta$.

Therefore, if $r_{j} \leq \rho\left(x_{j}\right) / 4$, then

$$
\left\|\left(b(x)-b_{B_{j}}\right) \mathscr{R}^{H} a_{j}(x) \chi_{\left(8 B_{j}\right)^{c}}(x)\right\|_{L^{1}\left(\mathbb{R}^{n}\right)} \leq C[b]_{\theta} .
$$

Thus, we have

$$
\begin{aligned}
\left|\left\{x \in \mathbb{R}^{n}:\left|A_{i}(x)\right|>\frac{\lambda}{4}\right\}\right| & \leq \frac{C}{\lambda}\left\|A_{i}(x)\right\|_{L^{1}} \\
& \leq \frac{C[b]_{\theta}}{\lambda} \sum_{j=-\infty}^{\infty}\left|\lambda_{j}\right|, \quad i=1,2,3 .
\end{aligned}
$$

Note that

$$
\begin{aligned}
\left\|\left(b-b_{B_{j}}\right) a_{j}\right\|_{L^{1}} & \leq\left(\int_{B_{j}}\left|b(x)-b_{B}\right|^{q^{\prime}} d x\right)^{1 / q^{\prime}}\left\|a_{j}\right\|_{L^{q}} \\
& \leq\left(\frac{1}{\mu\left(B_{j}\right)} \int_{B_{j}}\left|b(x)-b_{B}\right|^{q^{\prime}} d x\right)^{1 / q^{\prime}} \\
& \leq C[b]_{\theta}\left(1+\frac{r_{j}}{\rho\left(x_{j}\right)}\right)^{\theta^{\prime}} \\
& \leq C[b]_{\theta},
\end{aligned}
$$

where $r_{j}<\rho\left(x_{j}\right)$. get

By the weak $(1,1)$ boundedness of $\mathscr{R}^{H}$ (cf. Lemma 19), we

$$
\begin{aligned}
\left|\left\{x \in \mathbb{R}^{n}:\left|A_{4}(x)\right|>\frac{\lambda}{4}\right\}\right| & \leq \frac{C}{\lambda}\left\|\sum_{j=-\infty}^{\infty} \lambda_{j}\left(b-b\left(x_{j}\right)\right) a_{j}\right\|_{L^{1}} \\
& \leq \frac{C[b]_{\theta}}{\lambda} \sum_{j=-\infty}^{\infty}\left|\lambda_{j}\right| .
\end{aligned}
$$


Therefore,

$$
\begin{aligned}
& \left|\left\{x \in \mathbb{R}^{n}:|[b, T] f(x)|>\frac{\lambda}{4}\right\}\right| \\
& \leq C \sum_{i=1}^{4}\left|\left\{x \in \mathbb{R}^{n}:\left|A_{i}(x)\right|>\frac{\lambda}{4}\right\}\right| \\
& \leq \frac{C[b]_{\theta}}{\lambda} \sum_{j=-\infty}^{\infty}\left|\lambda_{j}\right| \\
& \leq \frac{C[b]_{\theta}}{\lambda}\|f\|_{H_{L}^{1}} .
\end{aligned}
$$

This completes the proof of Theorem 6 .

\section{Another Case}

In this section, we obtain same results for the commutator $\mathscr{R}^{H}$ if we impose another condition on $V$. Via Corollaries 15 and 16 in Section 2 and Theorems 1.2 and 1.3 in [23], we obtain the following theorems.

Theorem 27. Suppose that $V \in B_{s}$ for some $s>n,|\nabla V| \in B_{s_{1}}$ for some $s_{1}>n / 2$, and $|\nabla V|$ satisfies (32). Let $b \in B M O_{\infty}(\rho)$. The commutator $\mathscr{R}_{b}^{H}$ is bounded on $L^{p}\left(\mathbb{R}^{n}\right)$ for $1<p<s_{1}$.

Theorem 28. Suppose that $V \in B_{s}$ for some $s>n,|\nabla V| \in B_{s_{1}}$ for some $s_{1}>n / 2$, and $|\nabla V|$ satisfies (32). Let $b \in B M O_{\infty}(\rho)$. Then, for any $\lambda>0$,

$$
\begin{array}{r}
\left|\left\{x \in \mathbb{R}^{n}:\left|\mathscr{R}_{b}^{H}(f)(x)\right|>\lambda\right\}\right| \lesssim \frac{[b]_{\theta}}{\lambda}\|f\|_{H_{L}^{1}\left(\mathbb{R}^{n}\right)}, \\
\forall f \in H_{L}^{1}\left(\mathbb{R}^{n}\right) .
\end{array}
$$

Namely, the commutator $\mathscr{R}_{b}^{H}$ is bounded from $H_{L}^{1}\left(\mathbb{R}^{n}\right)$ into $L_{\text {weak }}^{1}\left(\mathbb{R}^{n}\right)$.

Remark 29. Following Remark 5 in [22], we know that if $V$ is a non-negative polynomial, condition (32) holds true. Furthermore, we know that if $V(x)=|p(x)|^{\alpha}$, where $p(x)$ is a polynomial and $\alpha>0$, condition (32) holds true (see Remark 6 in [24]).

\section{Examples}

In this section, we give some examples for the potentials which can satisfy the assumption in Theorems 4 and 6. We always assume that $n=3$ throughout this section. Denote the norm of $\mathbb{R}^{3}$ by $|x|=\left(x_{1}^{2}+x_{2}^{2}+x_{3}^{2}\right)^{1 / 2}$.

Example 1. Let

$$
V(x)=1+|x|=1+\left(x_{1}^{2}+x_{2}^{2}+x_{3}^{2}\right)^{1 / 2} \text {. }
$$

Following [25], we know that if $P(x)$ is a polynomial of degree $k$ and $\alpha>0$, then $V(x)=|P(x)|^{\alpha}$ belongs to $B_{\infty}\left(\mathbb{R}^{3}\right)$.
For $1<p<\infty$, it is easy to see that $B_{\infty}\left(\mathbb{R}^{3}\right) \subseteq B_{p}\left(\mathbb{R}^{3}\right)$. Moreover, it follows from (0.14) in [26] that

$$
m(x, V) \sim \sum_{|\beta| \leq k}\left|\partial_{x}^{\beta} P(x)\right|^{\alpha /(\alpha|\beta|+2)} .
$$

Therefore, $V(x)=1+|x|=1+\left(x_{1}^{2}+x_{2}^{2}+x_{3}^{2}\right)^{1 / 2} \in B_{\infty}\left(\mathbb{R}^{3}\right)$. If $V_{1}(x) \lesssim V_{2}(x)$, then

$$
\begin{aligned}
\frac{1}{m\left(x, V_{2}\right)} & \doteq \sup _{r>0}\left\{r: \frac{1}{r} \int_{B(x, r)} V_{2}(y) d y \leq 1\right\} \lesssim \frac{1}{m\left(x, V_{1}\right)} \\
& \doteq \sup _{r>0}\left\{r: \frac{1}{r} \int_{B(x, r)} V_{1}(y) d y \leq 1\right\} .
\end{aligned}
$$

Thus,

$$
\begin{gathered}
\nabla V(x)=\left(\frac{x_{1}}{\left(x_{1}^{2}+x_{2}^{2}+x_{3}^{2}\right)^{1 / 2}}, \frac{x_{2}}{\left(x_{1}^{2}+x_{2}^{2}+x_{3}^{2}\right)^{1 / 2}},\right. \\
\left.\frac{x_{3}}{\left(x_{1}^{2}+x_{2}^{2}+x_{3}^{2}\right)^{1 / 2}}\right) .
\end{gathered}
$$

Therefore, $|\nabla V(x)|=1$. Clearly, $|\nabla V(x)| \in B_{\infty}\left(\mathbb{R}^{3}\right)$. So, $V(x) \geq|\nabla V(x)|$. Therefore, $\rho(x) \lesssim \rho_{1}(x)$. Also, since $V(x) \geq$ 1 , then $\rho(x) \leqslant 1$. Then, the potential $V(x)=1+|x|=1+$ $\left(x_{1}^{2}+x_{2}^{2}+x_{3}^{2}\right)^{1 / 2}$ satisfies the assumption of Theorems 4 and 6.

Example 2. Let $V(x)=1+|x|^{3}=1+\left(x_{1}^{2}+x_{2}^{2}+x_{3}^{2}\right)^{3 / 2}$. By the previous argument, we conclude that $V \in B_{\infty}\left(\mathbb{R}^{n}\right)$.

Then,

$$
\begin{gathered}
\nabla V(x)=\left(3\left(x_{1}^{2}+x_{2}^{2}+x_{3}^{2}\right)^{1 / 2} x_{1}, 3\left(x_{1}^{2}+x_{2}^{2}+x_{3}^{2}\right)^{1 / 2} x_{2}\right. \\
\left.3\left(x_{1}^{2}+x_{2}^{2}+x_{3}^{2}\right)^{1 / 2} x_{3}\right) .
\end{gathered}
$$

Thus, $|\nabla V(x)|=3|x|^{2}=3\left(x_{1}^{2}+x_{2}^{2}+x_{3}^{2}\right)$. Clearly, $|\nabla V(x)| \epsilon$ $B_{\infty}\left(\mathbb{R}^{3}\right)$. From (99), we know that $m(x, V) \sim 1+|x|^{3}$ and $m(x,|\nabla V|) \sim 1+|x|$. Therefore, $\rho(x) \lesssim \rho_{1}(x)$. Also, since $V(x) \geq 1$, then $\rho(x) \lesssim 1$. Then, the potential $V(x)=1+|x|=$ $1+\left(x_{1}^{2}+x_{2}^{2}+x_{3}^{2}\right)^{3 / 2}$ satisfies the assumption of Theorems 4 and 6 .

Example 3. Let $V(x)=1+\left(x_{1}^{4}+x_{2}^{4}+x_{3}^{4}\right)^{1 / 2}$. By the previous argument, we conclude that $V \in B_{\infty}\left(\mathbb{R}^{n}\right)$.

Then,

$$
\begin{gathered}
\nabla V(x)=\left(2\left(x_{1}^{4}+x_{2}^{4}+x_{3}^{4}\right)^{-1 / 2} x_{1}^{3}, 2\left(x_{1}^{4}+x_{2}^{4}+x_{3}^{4}\right)^{-1 / 2} x_{2}^{3},\right. \\
\left.2\left(x_{1}^{4}+x_{2}^{4}+x_{3}^{4}\right)^{-1 / 2} x_{3}^{3}\right) .
\end{gathered}
$$

Thus,

$$
|\nabla V(x)|=2\left(\frac{x_{1}^{6}+x_{2}^{6}+x_{3}^{6}}{x_{1}^{4}+x_{2}^{4}+x_{3}^{4}}\right)^{1 / 2}
$$


From (99), we know that $m(x, V) \sim 1+|x|$. Since

$$
\begin{aligned}
& \left(x_{1}^{6}+x_{2}^{6}+x_{3}^{6}\right) \sim\left(x_{1}^{2}+x_{2}^{2}+x_{3}^{2}\right)^{3}, \\
& \left(x_{1}^{4}+x_{2}^{4}+x_{3}^{4}\right) \sim\left(x_{1}^{2}+x_{2}^{2}+x_{3}^{2}\right)^{2},
\end{aligned}
$$

then

$$
|\nabla V(x)| \sim\left(x_{1}^{2}+x_{2}^{2}+x_{3}^{2}\right)^{1 / 2} .
$$

Thus, $|\nabla V(x)| \in B_{\infty}\left(\mathbb{R}^{3}\right)$. From (99), we know that $m(x$, $|\nabla V|) \sim 1+|x|^{1 / 2}$. Therefore, $\rho(x) \lesssim \rho_{1}(x)$. Also, since $V(x) \geq 1$, then $\rho(x) \leqslant 1$. Then, the potential $V(x)=1+$ $\left(x_{1}^{4}+x_{2}^{4}+x_{3}^{4}\right)^{1 / 2}$ satisfies the assumption of Theorems 4 and 6.

\section{Acknowledgments}

This paper is supported by Research Fund for the Doctoral Program of Higher Education of China under Grant (no. 20113108120001), the Shanghai Leading Academic Discipline Project (J50101), the National Natural Science Foundation of China under Grant (no. 10901018), and the Fundamental Research Funds for the Central Universities. we use the fact that $\widetilde{\mathscr{R}}^{H}$ is bounded on $L^{p}\left(\mathbb{R}^{n}\right)$

\section{References}

[1] R. R. Coifman, R. Rochberg, and G. Weiss, "Factorization theorems for Hardy spaces in several variables," Annals of Mathematics, vol. 103, no. 3, pp. 611-635, 1976.

[2] S. Janson, "Mean oscillation and commutators of singular integral operators," Arkiv för Matematik, vol. 16, no. 2, pp. 263-270, 1978.

[3] S. Z. Lu, Q. Wu, and D. Yang, "Boundedness of commutators on Hardy type spaces," Science in China A, vol. 45, no. 8, pp. 984997, 2002.

[4] M. Bramanti and M. C. Cerutti, "Commutators of singular integrals on homogeneous spaces," Unione Matematica Italiana $B$, vol. 10, no. 4, pp. 843-883, 1996.

[5] X. T. Duong and L. X. Yan, "Commutators of BMO functions and singular integral operators with non-smooth kernels," Bulletin of the Australian Mathematical Society, vol. 67, no. 2, pp. 187-200, 2003.

[6] G. Pradolini and O. Salinas, "Commutators of singular integrals on spaces of homogeneous type," Czechoslovak Mathematical Journal, vol. 57, no. 1, pp. 75-93, 2007.

[7] J. Zhong, Harmornic analysis for some Schrödinger type operators [Ph.D. thesis], Princeton University, Princeton, NJ, USA, 1993.

[8] Z. W. Shen, " $L^{p}$ estimates for Schrödinger operators with certain potentials," Annales de l'institut Fourier, vol. 45, no. 2, pp. 513546, 1995.

[9] H.-Q. Li, "Estimations $L^{p}$ des opérateurs de Schrödinger sur les groupes nilpotents," Journal of Functional Analysis, vol. 161, no. 1, pp. 152-218, 1999.

[10] X. T. Duong, E. M. Ouhabaz, and L. Yan, "Endpoint estimates for Riesz transforms of magnetic Schrödinger operators," Arkiv för Matematik, vol. 44, no. 2, pp. 261-275, 2006.
[11] X. T. Duong and L. X. Yan, "Commutators of BMO functions and singular integral operators with non-smooth kernels," Bulletin of the Australian Mathematical Society, vol. 67, no. 2, pp. 187-200, 2003.

[12] Z. H. Guo, P. T. Li, and L. Z. Peng, " $L^{p}$ boundedness of commutators of Riesz transform associated to Schrodinger operator," Journal of Mathematical Analysis and Applications, vol. 241, no. 1, pp. 421-432, 2008.

[13] Y. Liu and J. F. Dong, "Some estimates of higher order Riesz transform related to Schrödinger type operators," Potential Analysis, vol. 32, no. 1, pp. 41-55, 2010.

[14] D. Yang, D. Yang, and Y. Zhou, "Endpoint properties of localized Riesz transforms and fractional integrals associated to Schrödinger operators," Potential Analysis, vol. 30, no. 3, pp. 271-300, 2009.

[15] P. T. Li and L. Z. Peng, "Endpoint estimates for commutators of Riesz transforms associated with Schrödinger operators," Bulletin of the Australian Mathematical Society, vol. 82, no. 3, pp. 367-389, 2010.

[16] L. Tang and J. F. Dong, "Boundedness for some Schrödinger type operators on Morrey spaces related to certain nonnegative potentials," Journal of Mathematical Analysis and Applications, vol. 355, no. 1, pp. 101-109, 2009.

[17] B. Bongioanni, E. Harboure, and O. Salinas, "Commutators of Riesz transforms related to Schrödinger operators," The Journal of Fourier Analysis and Applications, vol. 17, no. 1, pp. 115-134, 2011.

[18] J. F. Dong and Y. Liu, "The higher order Riesz transform and BMO type space associated to Schrödinger operators," Mathematische Nachrichten, vol. 285, no. 4, pp. 486-496, 2012.

[19] Y. Liu, "Commutators of BMO functions and degenerate Schrödinger operators with certain nonnegative potentials," Monatshefte für Mathematik, vol. 165, no. 1, pp. 41-56, 2012.

[20] J. Dziubański and J. Zienkiewicz, "Hardy space $H^{1}$ associated to Schrödinger operator with potential satisfying reverse Hölder inequality," Revista Matemática Iberoamericana, vol. 15, no. 2, pp. 279-296, 1999.

[21] D. Yang and Y. Zhou, "Localized Hardy spaces $H^{1}$ related to admissible functions on RD-spaces and applications to Schrödinger operators," Transactions of the American Mathematical Society, vol. 363, no. 3, pp. 1197-1239, 2011.

[22] S. Sugano, " $L^{p}$ estimates for some Schrödinger type operators and a Calderón-Zygmund operator of Schrödinger type," Tokyo Journal of Mathematics, vol. 30, no. 1, pp. 179-197, 2007.

[23] Y. Liu, J. Z. Huang, and J. F. Dong, "Commutators of CalderonZygmund operators related to admissible functions on spaces of homogeneous type and applications to Schrodinger operators," Science China Mathematics, 2012.

[24] K. Kurata and S. Sugano, "Estimates of the fundamental solution for magnetic Schrödinger operators and their applications," The Tohoku Mathematical Journal, vol. 52, no. 3, pp. 367-382, 2000.

[25] C. L. Fefferman, "The uncertainty principle," Bulletin of the American Mathematical Society, vol. 9, no. 2, pp. 129-206, 1983.

[26] Z. W. Shen, "Estimates in $L^{p}$ for magnetic Schrödinger operators," Indiana University Mathematics Journal, vol. 45, no. 3, pp. 817-841, 1996. 


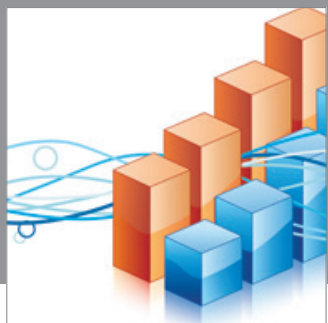

Advances in

Operations Research

mansans

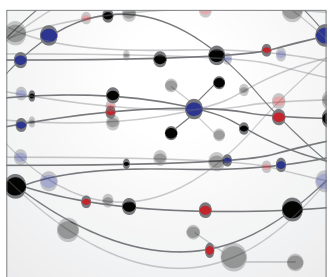

The Scientific World Journal
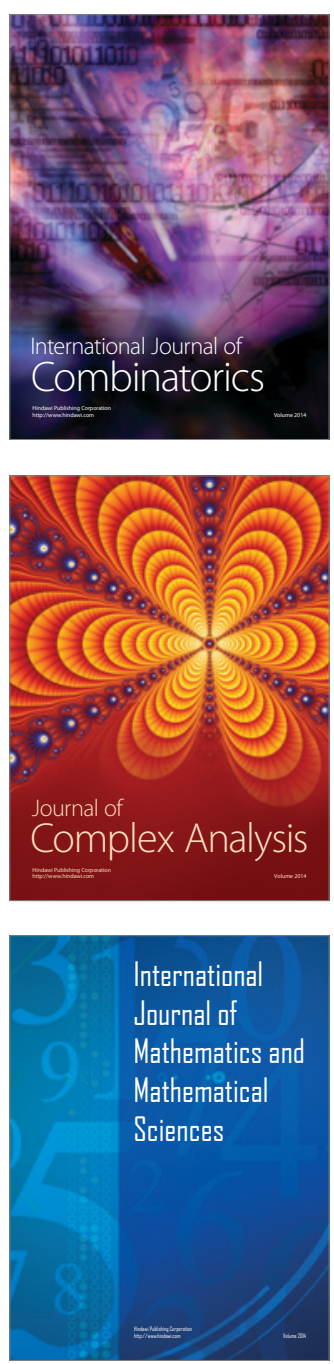
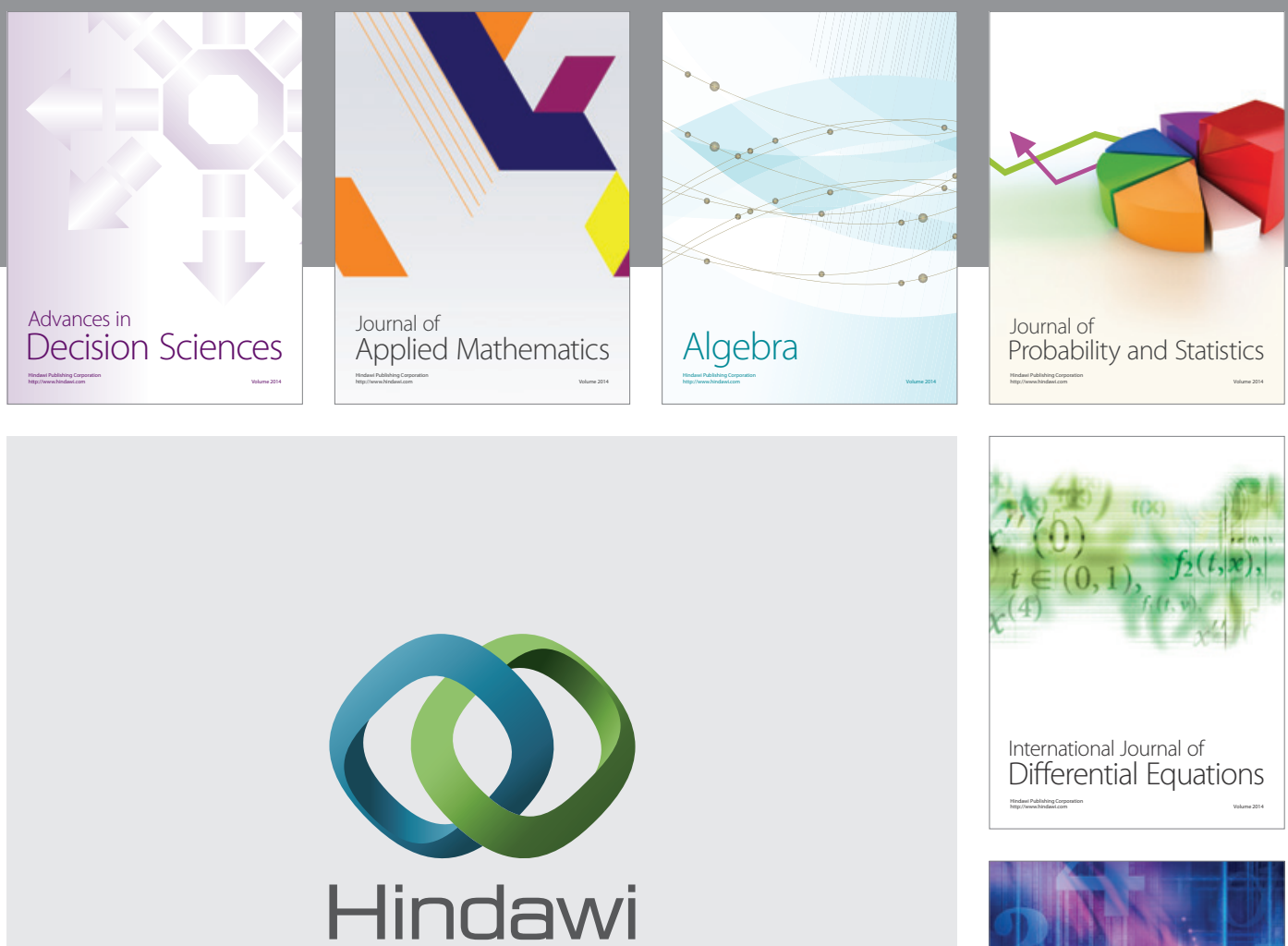

Submit your manuscripts at http://www.hindawi.com
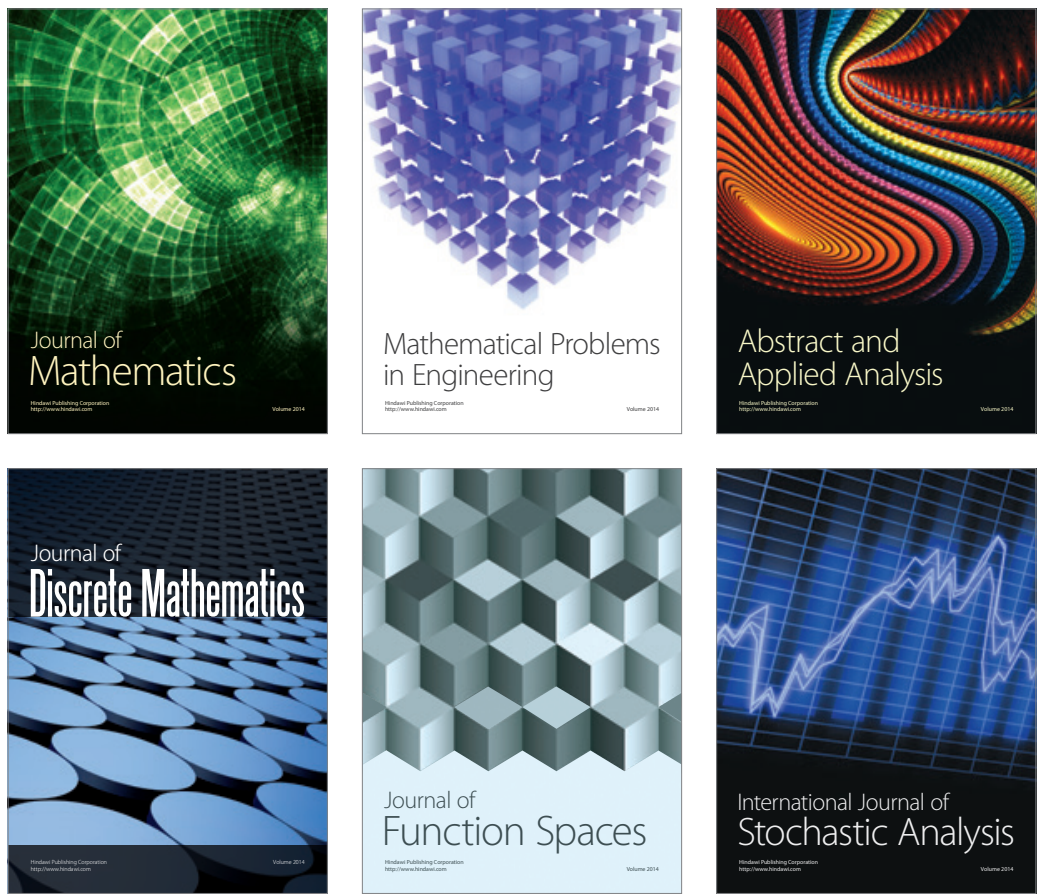

Journal of

Function Spaces

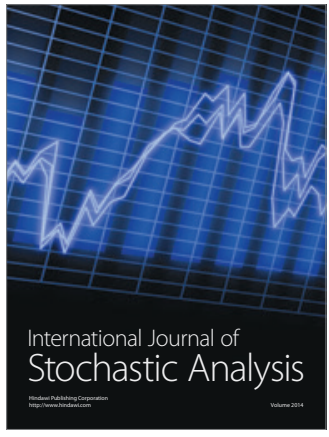

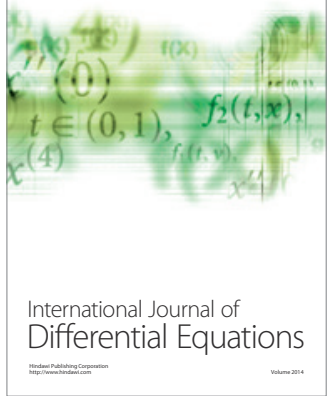
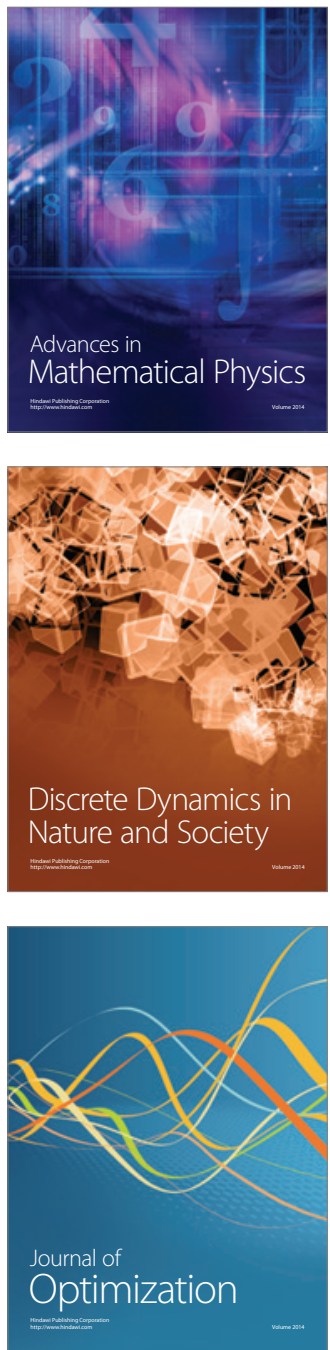